\title{
Visual Recognition Is Heralded by Shifts in Local Field Potential Oscillations and Inhibitory Networks in Primary Visual Cortex
}

\author{
Dustin J. Hayden, ${ }^{1 \star}$ Daniel P. Montgomery, ${ }^{1 \star}$ Samuel F. Cooke, ${ }^{2}$ and ${ }^{\oplus}$ Mark F. Bear $^{1}$ \\ ${ }^{1}$ Picower Institute for Learning and Memory, Department of Brain and Cognitive Sciences, Massachusetts Institute of Technology, Cambridge, \\ Massachusetts 02139, and ${ }^{2}$ Medical Research Council Centre for Neurodevelopmental Disorders, Department of Basic and Clinical Neurosciences, \\ Institute of Psychiatry, Psychology, and Neuroscience, King's College London, London SE5 9RT, England
}

Learning to recognize and filter familiar, irrelevant sensory stimuli eases the computational burden on the cerebral cortex. Inhibition is a candidate mechanism in this filtration process, and oscillations in the cortical local field potential (LFP) serve as markers of the engagement of different inhibitory neurons. We show here that LFP oscillatory activity in visual cortex is profoundly altered as male and female mice learn to recognize an oriented grating stimulus-low-frequency $(\sim 15 \mathrm{~Hz}$ peak) power sharply increases, whereas high-frequency $(\sim 65 \mathrm{~Hz}$ peak) power decreases. These changes report recognition of the familiar pattern as they disappear when the stimulus is rotated to a novel orientation. Two-photon imaging of neuronal activity reveals that parvalbumin-expressing inhibitory neurons disengage with familiar stimuli and reactivate to novelty, whereas somatostatin-expressing inhibitory neurons show opposing activity patterns. We propose a model in which the balance of two interacting interneuron circuits shifts as novel stimuli become familiar.

Key words: beta oscillations; gamma oscillations; long-term potentiation; novelty detection; stimulus-selective response potentiation; visual recognition memory

\section{Significance Statement}

Habituation, familiarity, and novelty detection are fundamental cognitive processes that enable organisms to adaptively filter meaningless stimuli and focus attention on potentially important elements of their environment. We have shown that this process can be studied fruitfully in the mouse primary visual cortex by using simple grating stimuli for which novelty and familiarity are defined by orientation and by measuring stimulus-evoked and continuous local field potentials. Altered eventrelated and spontaneous potentials, and deficient habituation, are well-documented features of several neurodevelopmental psychiatric disorders. The paradigm described here will be valuable to interrogate the origins of these signals and the meaning of their disruption more deeply.

Received Feb. 22, 2021; revised May 18, 2021; accepted May 19, 2021.

Author contributions: M.F.B., D.J.H., D.P.M., and S.F.C. designed research; D.J.H. and D.P.M. performed research; D.J.H. and D.P.M. analyzed data; M.F.B., D.J.H., and S.F.C. wrote the paper.

Support was provided by the National Institutes of Health (R01EY023037), the Picower Institute Innovation Fund, the Picower Young Faculty Support Fund, and a National Science Foundation Graduate Research Fellowship (D.J.H.). S.F.C. was supported by the Wellcome Trust (207727/Z/17/Z) and the Biotechnology and Biological Sciences Research Council (BB/S008276/1).We thank Arnold Heynen, Nina Palisano, Jessica Buckey, Athene Wilson-Glover, Kiki Chu, and Erin Hickey. We also thank Dr. Robert W. Komorowski for initiating and encouraging this project.

${ }^{*}$ D.J.H. and D.P.M. contributed equally to this work.

The authors declare no competing financial interests.

Correspondence should be addressed to Mark F. Bear at mbear@mit.edu.

https://doi.org/10.1523/JNEUROSCI.0391-21.2021

Copyright $\odot 2021$ Hayden, Montgomery et al.

This is an open-access article distributed under the terms of the Creative Commons Attribution 4.0 International license, which permits unrestricted use, distribution and reproduction in any medium provided that the original work is properly attributed.

\section{Introduction}

The awake brain receives a steady stream of sensory stimuli. Distinguishing novel, potentially relevant stimuli from familiar, irrelevant stimuli is essential for the dedication of energy and attention to only those elements of the environment that may be salient for survival. Previous studies have described an electrophysiological signature of long-term recognition memory within the primary visual cortex (V1) of mice that is highly selective for stimulus attributes, such as orientation (Frenkel et al., 2006; Cooke and Bear, 2010). Over days of repeated presentation of a simple, phase-reversing sinusoidal grating stimulus, the magnitude of visually evoked potentials (VEPs) recorded in layer 4 of $\mathrm{V} 1$ in awake, head-fixed mice significantly increases. We refer to this process as stimulus-selective response plasticity (SRP). Similar phenomena have been reported by others (Aton et al., 2014; Kaneko and Stryker, 2014; Kaneko et al., 2017; Kissinger et al., 2018). 
Much headway has been made in understanding the requirements for SRP. Disruption of SRP by treatments local to V1, notably including manipulations of NMDA receptor (NMDAR) function and AMPA receptor trafficking in principal cells, had suggested involvement of the mechanisms of long-term potentiation (LTP) of feedforward excitatory synapses (Frenkel et al., 2006; Cooke et al., 2015). However, more recent studies show that SRP (1) is not supported by plasticity at excitatory layer 4 synapses (Cooke and Bear, 2014; Fong et al., 2020) and (2) depends on the activity of parvalbumin-expressing $(\mathrm{PV}+)$ neurons in V1 (Kaplan et al., 2016). Given the extensive evidence that $\mathrm{PV}+$ inhibitory neurons contribute to gamma oscillations $(\geq 40 \mathrm{~Hz})$ in the cortex (Cardin et al., 2009; Korotkova et al., 2010; Carlén et al., 2012; Gonzalez-Burgos and Lewis, 2012; Lewis et al., 2012; Kuki et al., 2015; Jadi et al., 2016; Polepalli et al., 2017), in the current study we sought to understand how the local field potential (LFP) and PV + cell activity in layer 4 are influenced by stimulus familiarity and how these changes evolve over time.

We found that exposure of mice to a novel visual stimulus elicits high-frequency $(\sim 65 \mathrm{~Hz})$ oscillations in the LFP and increases PV + cell activity measured using two-photon (2-p) calcium imaging. With repeated viewing over days, the now-familiar stimulus elicited reduced high-frequency power in the LFP with a corresponding decrease in PV + cell activity, as well as a sharp increase in low-frequency $(\sim 15 \mathrm{~Hz} ; \beta)$ oscillations. The observed increase in oscillations at this frequency is consistent with the hypothesis that familiar stimuli recruit a population of somatostatin-expressing (SOM+) interneurons (Kuki et al., 2015) as has been observed in auditory cortex following longterm habituation with passive sound exposure (Kato et al., 2015). Indeed, 2-p calcium imaging in SOM+ cells in V1 revealed an increase in their activity during familiar stimulus viewing. These signatures of familiar stimulus recognition did not appear immediately on stimulus onset but developed rapidly within the first few seconds of visual stimulation. Our findings significantly advance the understanding of how visual recognition memory is expressed at the circuit level and demonstrate how stimulus familiarity imposes oscillatory activity on cerebral cortex.

\section{Materials and Methods}

Mice. All procedures adhered to the guidelines of the National Institutes of Health and were approved by the Committee on Animal Care at Massachusetts Institute of Technology. For local field potential experiments, we used male and female mice on a C57BL/6 background (Charles River Laboratories). For calcium imaging experiments, we used male and female PV-Cre mice (B6.129P2-Pvalb ${ }^{\text {tm1(cre)Arbr} / J ; ~ c a t a l o g ~}$ \#017320, The Jackson Laboratory; RRID:IMSR_JAX:017320) and SOM-

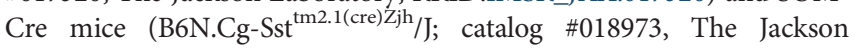
Laboratory; RRID:IMSR_JAX:018973). The familiar-novel differences reported in this study did not differ qualitatively by sex, so both were combined in agreement with previous studies (Fong et al., 2020). Animals were housed in groups of 2-5 same-sex littermates after weaning at postnatal day 21 (P21). They had access to food and water ad libitum and were maintained on a $12 \mathrm{~h}$ light-dark cycle.

Surgery. For local field potential experiments, young adult C57BL/6 mice (P26-P52) were first injected with $0.1 \mathrm{mg} / \mathrm{kg}$ Buprenex subcutaneously (s.c.) to provide analgesia. Induction of anesthesia was achieved via inhalation of isoflurane (3\% in oxygen) and thereafter maintained via inhalant isoflurane ( $\sim 1-2 \%$ in oxygen). Before surgical incision, the head was shaved and the scalp cleaned with povidone-iodine (10\% w/v) and ethanol $(70 \% \mathrm{v} / \mathrm{v})$. The scalp was resected, and the skull surface was scored. A steel head post was affixed to the skull (anterior to bregma) with cyanoacrylate glue. Small burr holes were drilled above both hemispheres of binocular V1 (3.0 mm lateral of lambda). Tapered 300$500 \mathrm{k} \Omega$ tungsten recording electrodes (FHC), $75 \mu \mathrm{m}$ in diameter at their widest point, were implanted in each hemisphere, $450 \mu \mathrm{m}$ below the cortical surface. Silver wire (A-M Systems) reference electrodes were placed over the left frontal cortex. Electrodes were secured using cyanoacrylate, and the skull was covered with dental cement. Nonsteroidal antiinflammatory drugs were administered on return to the home cage (meloxicam, $1 \mathrm{mg} / \mathrm{kg}$ s.c.). Signs of infection and discomfort were carefully monitored. Mice were allowed to recover for at least $48 \mathrm{~h}$ before head fixation.

For cranial window implantations for two-photon calcium imaging, adult PV-Cre or SOM-Cre mice (P43-P133) were anesthetized and prepared as described above. Following scalp incision, a lidocaine (1\%) solution was applied onto the periosteum, and the exposed area of skull gently scraped with a scalpel blade. Then, a $3 \mathrm{~mm}$ craniotomy was made over binocular V1. Adeno-associated virus containing the GCaMP7f gene (pGP-AAV9-syn-FLEX-jGCaMP7f-WPRE; catalog \#104488AAV9, Addgene) was loaded into a glass micropipette with a tip diameter of $40-50 \mu \mathrm{m}$ attached to a Nanoject II injection system (Drummond Scientific). The micropipette was then inserted into binocular V1 layer 4 at depths of 400 and $450 \mu \mathrm{m}$ below the pial surface, and $\sim 50 \mathrm{nl}$ of virus was delivered at each depth. Next, a sterile 3 -mm-round glass coverslip (CS-3R-0; Warner Instruments) was gently laid on top of the exposed dura mater. The coverslip was secured with cyanoacrylate glue, and a stainless-steel head post was attached to the skull. Once the glue had set, dental acrylic (C\&B Metabond Quick Adhesive Cement System) was mixed and applied throughout the exposed skull surface.

Visual stimulus delivery. Before stimulus delivery, mice were acclimated to head restraint in front of a gray screen for a $30 \mathrm{~min}$ session on each of two consecutive days. After acclimation, for the LFP, pupil, and movement experiments, mice were presented with 5 blocks of 100 phase reversals of an oriented grating stimulus phase reversing at $0.5 \mathrm{~Hz}$. They were shown this stimulus for six consecutive days. On day 7, they were shown both the familiar stimulus orientation as well as blocks of a novel stimulus offset $90^{\circ}$ from the novel orientation. Each stimulus block was preceded by a period of gray screen, a period of black screen, and another period of gray screen. Gray periods lasted 6 or $12 \mathrm{~s}$, and black periods lasted 10 or $20 \mathrm{~s}$, depending on the recording system. Discrete sections of gray- and black-screen viewing were time stamped for later normalization. After habituation for the calcium imaging experiments, mice were presented with 5 blocks of 120 phase reversals of an oriented grating stimulus phase reversing at $0.5 \mathrm{~Hz}$. They were shown this stimulus for four consecutive days. On day 5 , they were shown both the familiar stimulus orientation as well as blocks of a novel stimulus offset $90^{\circ}$ from the novel orientation. Each stimulus block was preceded by $30 \mathrm{~s}$ of gray screen. To keep head restraint to a minimum during calcium imaging experiments, only four blocks of each stimulus were used on day 5 . For all experiments, if more than one orientation was shown within a session, stimulus blocks were pseudorandomly interleaved so that three consecutive presentations of the same stimulus never occurred. Visual stimuli consisted of full-field, $100 \%$ contrast, sinusoidal gratings that were presented on a computer monitor. Visual stimuli were generated using custom software written in either $\mathrm{C}++$ for interaction with a VSG2/2 card (Cambridge Research Systems) or MATLAB (MathWorks) using the PsychToolbox extension (http://psychtoolbox.org) to control stimulus drawing and timing. Grating stimuli spanned the full range of monitor display values between black and white, with gamma correction to ensure constant total luminance in both gray-screen and patterned stimulus conditions.

In vivo electrophysiology experimental design and analysis. Electrophysiological recordings were conducted in awake, headrestrained mice. Recordings were amplified and digitized using the Recorder-64 system (Plexon) or the RHD Recording System (Intan Technologies). Two recording channels were dedicated to recording continuous local field potential from V1 in each implanted hemisphere. In a subset of experiments, an additional third recording channel was reserved for the piezoelectrical input carrying the forepaw movement. Local field potential was recorded from V1 with $1 \mathrm{kHz}$ sampling. On the Plexon system, we used a $500 \mathrm{~Hz}$ low-pass 
filter. On the Intan system, we used a $0.1 \mathrm{~Hz}$ high-pass and a $7.5 \mathrm{kHz}$ low-pass filter. Local field potential data and piezoelectric data were imported (see below, Importing and data cleaning), and the local field potential's spectral content was analyzed (see below, Spectral analysis). In a subset of LFP experiments, forepaw movement was analyzed (see below, Movement analysis). In a separate LFP experiment, pupil dilation was monitored (see below, Pupil analysis).

In vivo two-photon calcium imaging. Three to 4 weeks following craniotomy surgery, mice were habituated to the behavior restraint apparatus in front of a gray screen with the objective lens of the two-photon microscope positioned on the head plate for $30 \mathrm{~min}$ for two consecutive days before beginning their visual stimulus delivery. A Ti:sapphire laser (Coherent) was used for imaging at a wave length of $930 \mathrm{~nm}$. Photomultiplier tubes (Hamamatsu) and the objective lens $(20 \times, 0.95$ numerical aperture, XLUMPLFLN, Olympus) were used to detect fluorescence images. Calcium image recordings were triggered by time-locked transistor-transistor logic pulses generated from the USB-1208fs data acquisition device (Measurement Computing) using PrairieView and TriggerSync software (Bruker) and imaged at a frequency of $\sim 2.8 \mathrm{~Hz}$ at the depth of $\sim 350 \mu \mathrm{m}$ in $\mathrm{V} 1$. The size of the imaging field of view was $\sim 600 \times 600 \mu \mathrm{m}^{2}$ at $256 \times 256$ pixels.

Pupillometry. To track the pupil during head fixation, we used a Blackfly S USB3 camera (Teledyne FLIR) with a $1.0 \times$ lens (Edmund Optics). The left eye was illuminated with a $780 \mathrm{~nm}$ infrared LED light source (Thorlabs). A small tissue was placed over the light source to disperse luminance. Images were acquired at 20 frames per s during stimulus presentation, and each frame emitted a voltage signal into the RHD Recording System for later alignment with stimulus presentations. A subset of videos was used for training the top and bottom edge of the pupil on DeepLabCut (Mathis et al., 2018). All videos were evaluated with the trained network. The output of DeepLabCut includes the $x$ and $y$ coordinates of the top and bottom edge as well as the certainty of the location. Both were used in our analysis (see below, Importing and data cleaning, and Pupil analysis).

Importing and data cleaning. All analyses were conducted using custom MATLAB code and the Chronux toolbox (Bokil et al., 2010). Briefly, the local field potential from each channel was extracted and converted to microvolts. Data were then zero meaned and detrended using a $500 \mathrm{~ms}$ sliding window and a $0.1 \mathrm{~s}$ step size. A third-order Butterworth filter was used to notch frequencies between 58 and $62 \mathrm{~Hz}$. The average voltage of the first $10 \mathrm{~ms}$ after a phase reversal was subtracted from each individual trace to align them. For average VEPs, data were smoothed with a Gaussian spanning $20 \mathrm{~ms}$ using MATLAB's smooth data function. Piezoelectric data were zero meaned and rectified. Pupil edges that had $<100 \%$ certainty of location in terms of DeepLabCut output (see above, Pupillometry) were ignored, and a spline interpolation was used to recover the missing points. Qualitatively, these periods of uncertain pupil edge location occurred frequently during the black-screen and rarely during grayscreen or visual stimulus presentations.

Spectral analysis. Given that the visually evoked potential violates assumptions required for spectral analysis (namely second-order stationarity), we only analyzed the spectral activity between $400 \mathrm{~ms}$ and $2000 \mathrm{~ms}$ after a phase reversal. We computed the multitapered spectrogram of the local field potential using the Chronux toolbox (Bokil et al., 2010). The parameters used were the following: a $500 \mathrm{~ms}$ sliding window; a $100 \mathrm{~ms}$ step size, zero-padded to the second power; and five tapers with a time bandwidth product of three. We also computed the multitapered spectrum using the same parameters but including all data between $400 \mathrm{~ms}$ and $2000 \mathrm{~ms}$. To calculate the normalized spectrum/ spectrogram, we found the median spectrum/spectrogram of the animal's black screen and took $10^{\star} \log 10$ (stimulus_spectrum/median_black_spectrum). This is reported as a decibel (dB).

Concatenated spectrum analysis. Given the contamination by the visually evoked potential, we concatenated the normalized spectrums. This concatenated spectrum uses the multitapered spectrum of the period between 400 and $2000 \mathrm{~ms}$ after a phase reversal. Ordering these spectrums by their presentation number and representing power as a color generated the concatenated spectrum. For each presentation, we calculated the maximum power within the $10-30 \mathrm{~Hz}$ frequency band as well as the $60-80 \mathrm{~Hz}$ frequency band. By visual inspection, no changes were seen in the concatenated spectrum after 25 presentations, so we used the average of the maximum power for presentations $26-100$ as a metric to compare the first few presentations in our bootstrapping procedure. No significant difference is found between presentations after the 15th and the average of presentations 26-100, confirming that this data split was reasonable. Other splits were tried, and there were no qualitative differences in the resulting data.

Block onset spectral analysis. For this analysis, we only used the group of animals that had $6 \mathrm{~s}$ gray periods and $10 \mathrm{~s}$ black periods (see above, Visual stimulus delivery). The local field potential data within $12 \mathrm{~s}$ of block onset (both before and after) were extracted and separated into overlapping $400 \mathrm{~ms}$ chunks each with centers spaced $100 \mathrm{~ms}$ apart. For each chunk, we computed the normalized spectrum (see above, Spectral analysis). Transitions from black to gray, gray to stimulus, and between phase $0^{\circ}$ and phase $180^{\circ}$ will elicit a visually evoked potential. Thus, for each frequency within the normalized spectrum, we removed contaminated regions and interpolated between them using cubic interpolation. Specifically, we removed the chunks whose midpoints were between $100 \mathrm{~ms}$ before and $500 \mathrm{~ms}$ after a transition. The trailing edge (near 12s) was not included due to the edge artifacts of interpolation. Once we had the interpolated normalized spectrum, we found the maximum power in the $10-30 \mathrm{~Hz}$ frequency band and the $60-80 \mathrm{~Hz}$ frequency band for each chunk.

$P$-Episode analysis. P-Episode is a method that quantifies the fraction of time that oscillations exceed amplitude and duration thresholds (Caplan et al., 2001; van Vugt et al., 2007). We lightly adapted analysis software provided by Marieke van Vugt (University of Groningen, The Netherlands). Briefly, Morlet wavelets between 7 and $100 \mathrm{~Hz}$ with a wave number of 5 were used to extract spectral information from both black and stimulus periods. Then, to obtain an estimate of the background spectral activity, we fit the black spectrum with a linear regression in $\log -\log$ space and stored the mean power values at each frequency (using the trained regression parameters). This model has the form $\mathrm{A} / \mathrm{f}^{\alpha}$ and is commonly called pink or colored noise (Caplan et al., 2001; van Vugt et al., 2007). This was done for each black period and ultimately averaged to get one estimate of the background spectrum per animal. In line with default parameters, the power threshold was determined for each frequency as the 95th percentile of the chi-squared probability distribution with 2 degrees of freedom. The duration threshold was simply three cycles. Next, for each presentation of a stimulus, the above Morlet wavelets were used to extract spectral information. Finally, for each time point, it was determined whether a given frequency exceeded both the power and duration thresholds. If it exceeded both thresholds, the time point was in that oscillation. Otherwise, the time point was not in that oscillation. Herein, we report the percentage of time in an oscillation for each of the analyzed frequencies.

Correlations. We analyzed the correlation of VEP magnitude and LFP power in single trials. Because the VEP in response to each phase reversal is variable and can be obscured by ongoing voltage fluctuations, it had to exceed a threshold to be included in the analysis. For each animal, we computed the average activity during exposure to the gray screen between each block of stimuli. The gray period was sampled at the same frequency and duration as used for VEP analysis. The difference between the minimum of this average gray activity (within $100 \mathrm{~ms}$ of the sample onset) and the maximum of this average gray activity (any time after the minimum) was taken as our voltage threshold. Next, we computed the average VEP above threshold for all familiar and novel presentations to get the indices for the positive and negative peaks (regardless of stimulus). Using these indices, for each phase reversal, we calculated the magnitude of the difference between the positive and negative peaks. If this single-trial VEP magnitude was below the voltage threshold decided by the gray-screen period, that trial was discarded ( $\sim 19 \%$ of trials). We additionally eliminated the first presentation of each block as we were comparing the pre-phase reversal LFP to the VEP magnitude, and the first presentation's LFP would be during a gray screen.

For each phase reversal, we also calculated the normalized spectrum for the $400 \mathrm{~ms}$ leading into the phase reversal. Using this, we obtained 
A

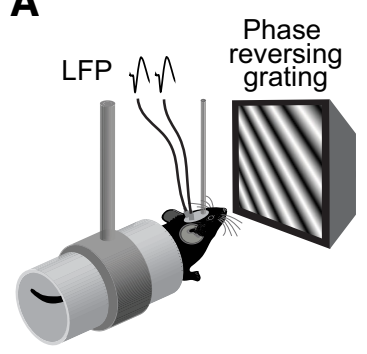

B

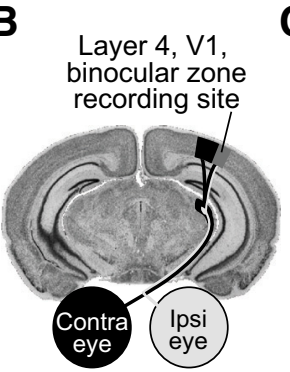

C

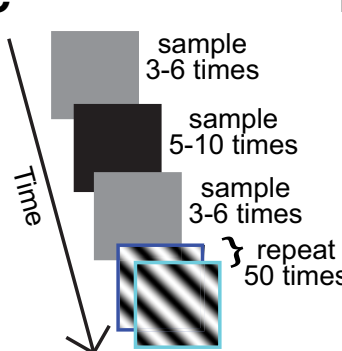

D

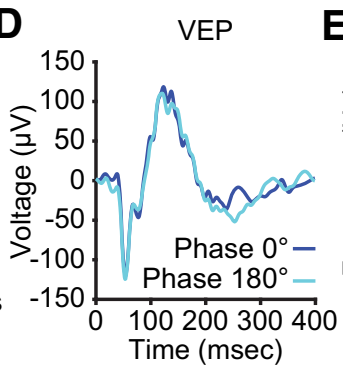

E

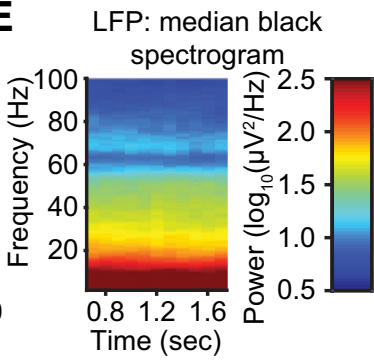
Time (sec)
F

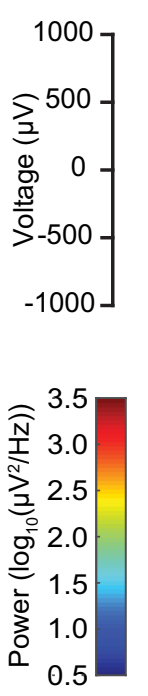

H

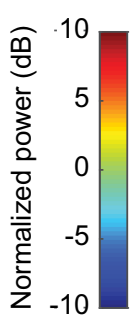

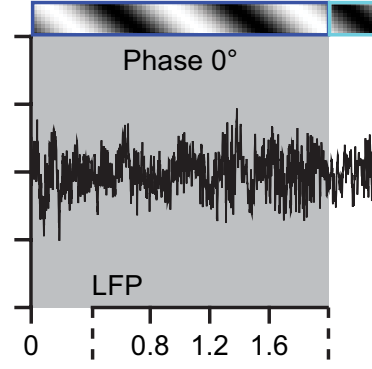

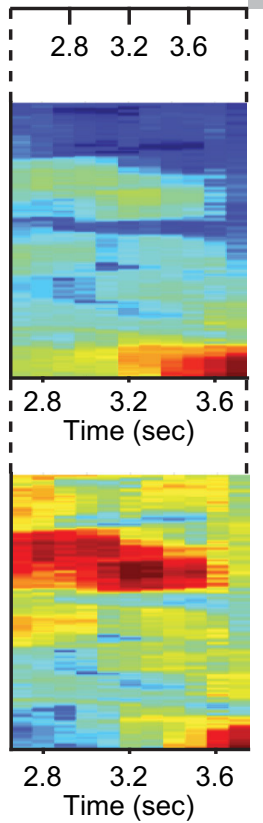

Phase $180^{\circ}$

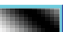

.

\section{(1)}
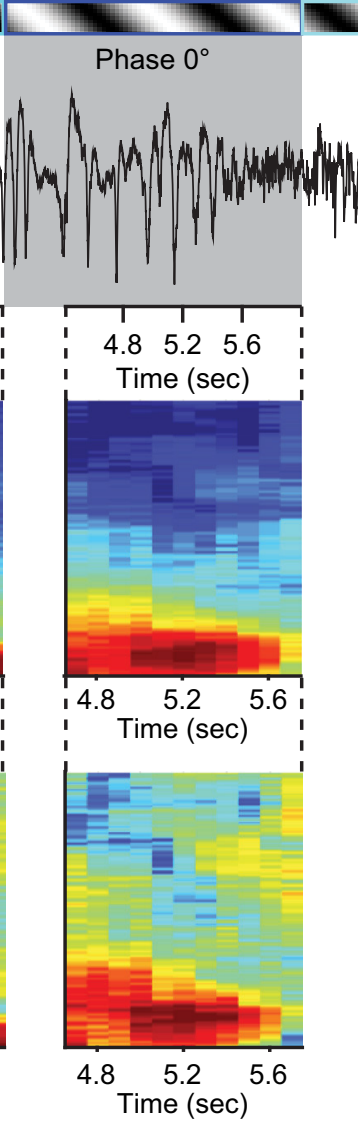

Phase $180^{\circ}$

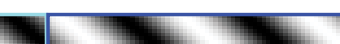



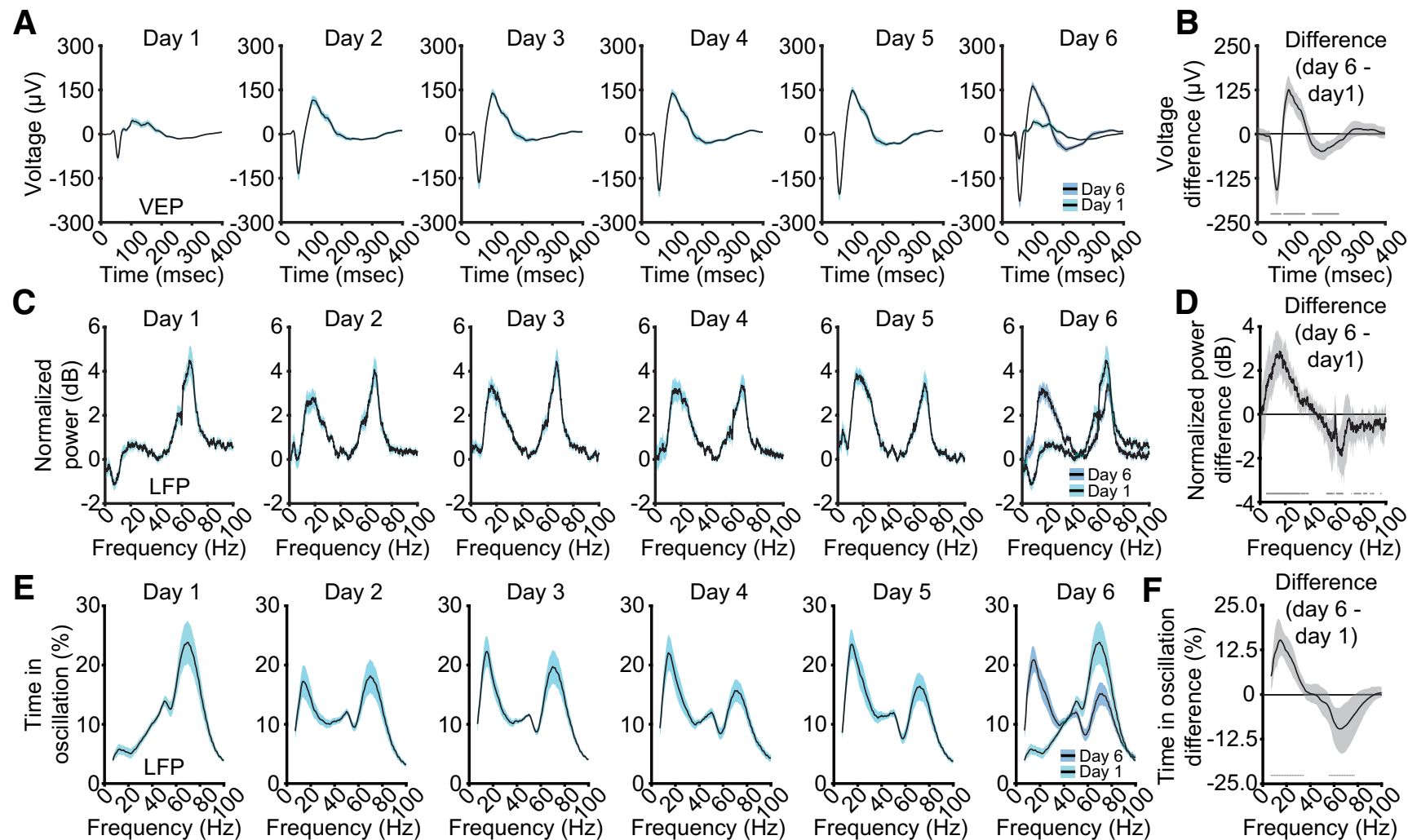

Frequency $(\mathrm{Hz})$

Frequency $(\mathrm{Hz})$

Difference
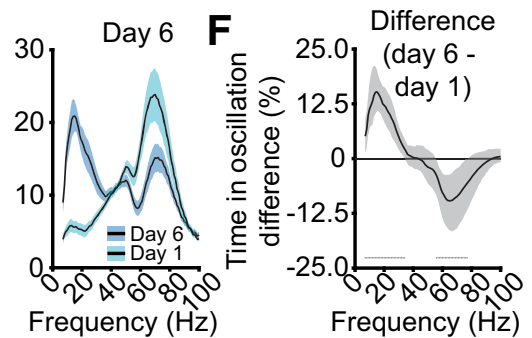

Figure 2. V1 VEPs and LFP oscillations are influenced by stimulus familiarity over days. $A$, Repeated presentation of the same stimulus orientation over six days increases the average VEP ( $n$ $=13$ ). $\boldsymbol{B}$, Nonparametric hierarchical bootstrapping results confirm that the VEP on day 1 (cyan) is significantly lower in magnitude than on day 6 (blue), despite the fact the stimulus has not changed. Solid line represents the median value, and the shaded region reflects the $99 \%$ confidence interval. C, Over those same six d in the same mice, low-frequency power increases and high-frequency power decreases. D, Nonparametric hierarchical bootstrapping results confirm that the spectrum on day 6 (blue) is significantly different from the spectrum on day 1 (cyan), despite the fact the stimulus has not changed. Solid line represents the median value, and the shaded region reflects the $99 \%$ confidence interval. $E$, Analysis of the amount of time the LFP shows sustained oscillatory activity for a given band. $\boldsymbol{F}$, Nonparametric hierarchical bootstrapping results of time differences. $\boldsymbol{B}, \boldsymbol{D}, \boldsymbol{F}$, Marks near the $x$-axis indicate the $99 \%$ confidence interval does not include zero (thus the difference is statistically significant). Averaged VEPs, spectrums, and P-Episode results are presented as mean \pm SEM.

Statistics. Most statistics were conducted with the nonparametric hierarchical bootstrap for multilevel data (Saravanan et al., 2020). Briefly, statistical comparisons were between two groups (with each animal belonging to both groups because of the within-animal experimental design). To begin the bootstrap process, mice were randomly selected with replacement from the population. For each randomly selected mouse, a number of random trials were selected with replacement from the mouse's group A data. Another random set of trials were selected with replacement from the mouse's group B data. These data were stored, and the process was repeated for each randomly selected animal. In some instances (see Figs. 4, 7, 8, 9, 12), blocks were randomly selected with replacement, and all trials or time points within that block were used. Once all data were randomly selected, the mean difference between the randomly selected samples of group A and group B was computed and stored. This entire bootstrap process was repeated 1000 times. Once all 1000 bootstraps had been completed, the bootstrapped differences were sorted from lowest to highest value. The 500th value was the median group difference, the 5th value was the lower bound of the $99 \%$ confidence interval (CI), and the 995th value was the upper bound of the $99 \%$ confidence interval. If the $99 \%$ confidence interval does not include zero, we report a statistically significant difference between group A and group B with a small marker below the corresponding data on the plot. To facilitate communication in the results section, we report the identity, median, and $99 \%$ confidence interval for the peak significant median difference above and below $50 \mathrm{~Hz}$, if it exists. In most figures, the colored plots are mean \pm SEM of all animals, and the gray plots to the right are the $99 \%$ bootstrapped confidence interval for those two groups. The only other statistical procedure was a two-sample Kolmogorov-Smirnov test on the data comprising the cumulative distribution functions in Figure 5.
Data availability. All data, code, and values are available for complete replication of the research on reasonable request to D.J.H or M.F.B.

\section{Results}

Layer 4 local field potential oscillations display variable frequency composition in V1 of awake, head-fixed mice

We acquired LFP data from electrodes chronically implanted within layer 4 of binocular V1 of C57BL/6 mice. Awake, headfixed mice viewed full field, $0.5 \mathrm{~Hz}$ phase-reversing sinusoidal grating stimuli separated into blocks of 100 phase reversals preceded by periods of gray and black screens (Fig. $1 A-C$ ). We used equally spaced time stamps to segment gray- and black-screen data into $2000 \mathrm{~ms}$ portions for further normalization and comparison. Under these conditions, we could average the stimulusevoked LFP waveform occurring within a $400 \mathrm{~ms}$ time window from the start of each phase reversal (representative mouse; Fig. $1 D$ ). This average VEP is the signal typically used to monitor the emergence of SRP (Frenkel et al., 2006; Cooke and Bear, 2010; Cooke et al., 2015; Kaplan et al., 2016). However, the continuous LFP signal reveals periodic changes from low-amplitude, highfrequency activity to high-amplitude, low-frequency activity (representative mouse; Fig. $1 F$ ). Because the portion of the recording containing the VEP violates second-order stationarity, a requirement for oscillatory analysis, we focused our analysis on the last $1600 \mathrm{~ms}$ of each $2000 \mathrm{~ms}$ presentation (Fig. 1F). This approach is consistent with previous work (Chalk et al., 2010; Zhou et al., 2016). The raw spectrogram for each phase reversal 
(Fig. 1F) shows that some time periods have strong low-frequency oscillations, whereas others have strong high-frequency oscillations (Fig. 1G). We chose not to normalize the raw signal to data from a gray-screen period because isoluminant gray screens elicit narrow-band oscillations at $60 \mathrm{~Hz}$ that emerge in the cortex but arise from a subcortical source (Saleem et al., 2017). Instead, we normalized the raw spectrogram to the median spectrogram generated during the black-screen presentation (Fig. 1E). The normalized spectrogram for each phase-reversal (Fig. $1 F)$ again shows periods of strong low-frequency oscillations in the $\alpha / \beta$ range (10$30 \mathrm{~Hz}$ ) and periods of high-frequency oscillations in $60-80 \mathrm{~Hz}$ range (Fig. $1 \mathrm{H}$ ). As there is unfortunate inconsistency in how the term "gamma" is used in the literature to describe oscillations in visual cortex (Chen et al., 2017; Veit et al., 2017), we have avoided use of this term to describe our findings. However, we note that activity in the $60-80 \mathrm{~Hz}$ range is conventionally defined as "high-gamma." The normalized spectral data are used throughout the remainder of this study.

\section{V1 oscillations are influenced by stimulus familiarity over days}

We investigated whether the frequency composition of the V1 LFP in layer 4 changes as a result of visual experience. We induced SRP by exposing mice to a phase-reversing stimulus at a single orientation each day for six consecutive days. As reported in previous studies ( (Frenkel et al., 2006; Cooke et al., 2015; Fong et al., 2020), the VEP magnitude increases over days (Fig. 2A). The VEP on day 6 is significantly larger than day 1 (Fig. $2 B$; median peak-to-peak difference: $285.05 \mu \mathrm{V}, 99 \% \mathrm{CI}=283.92,290.34 \mu \mathrm{V} ; n=13$ mice). In the same mice, on day 1 the normalized spectrum displayed strong high-frequency power (Fig. 2C). As the stimuli became familiar over subsequent days, high-frequency power diminished and low-frequency power increased. Comparing day 6 to day 1 showed day 6 had significantly more low-frequency power (Fig. $2 D$; peak: $15.50 \mathrm{~Hz}$, median difference, $2.88 \mathrm{~dB}, 99 \% \mathrm{CI}=1.99$, $3.67 \mathrm{~dB} ; n=13$ ) and less high-frequency power (Fig. $2 D$; peak: $64.82 \mathrm{~Hz}$, median difference: $-1.90 \mathrm{~dB}, 99 \% \mathrm{CI}=-3.17,-0.53$ $\mathrm{dB} ; n=13$ ). Thus, stimulus familiarity increases low-frequency power and decreases high-frequency power in layer 4 of V1.

The change in average spectrum power could be a result of a blanket increase in any given frequency at all times, more periods of sustained oscillatory activity, or some combination of both. We therefore measured the amount of time spent by the LFP within each frequency on each day. For our purposes, this was achieved with P-Episode (see above, Materials and Methods), a technique that only counts an oscillation as active if it surpasses both a power and duration threshold (Caplan et al., 2001; van Vugt et al., 2007). On day 1, the LFP spent more time exhibiting high-frequency oscillations than low (Fig. 2E). By day 6, time
B

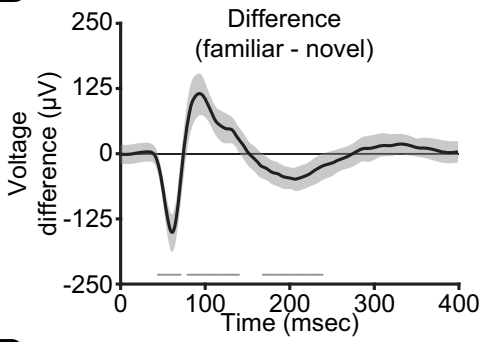

D
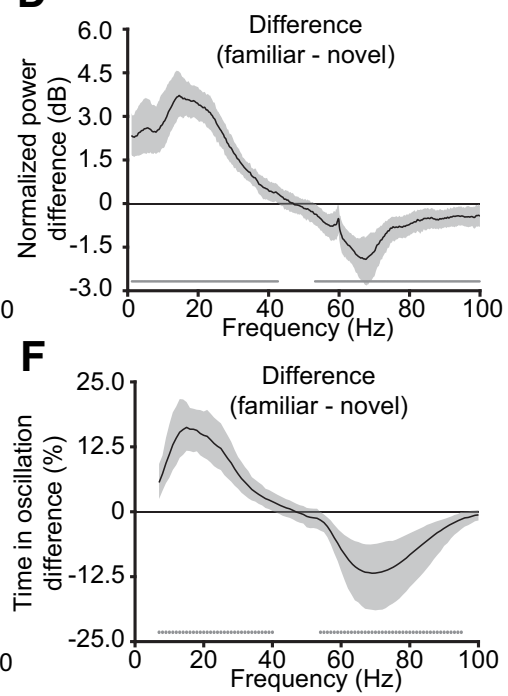

Figure 3. Experience-dependent changes in V1 VEPs and LFP oscillations are stimulus specific. $\boldsymbol{A}$, Presentation of a novel (red) stimulus elicits a smaller VEP than a familiar (blue) stimulus $(n=13)$. $\boldsymbol{B}$, Nonparametric hierarchical bootstrapping results confirm that the familiar VEP is significantly larger than the novel VEP. Solid line represents the median value, and (he same mice, presentation of a novel (red) stimulus increases Averaged VEPs, spectrums, and P-Episode results are presented as mean \pm SEM.

spent in high-frequency oscillations dropped, and time spent in low-frequency oscillations increased. Comparing day 6 with day 1 revealed that more time was spent in low-frequency oscillations on day 6 than day 1 (Fig. 2F; peak: $15.00 \mathrm{~Hz}$, median difference, $15.24 \%, 99 \% \mathrm{CI}=10.81,20.39 \% ; n=13$ ) and less time was spent in high-frequency oscillations on day 6 than day 1 (Fig. $2 F$; peak: $65.00 \mathrm{~Hz}$, median difference: $-9.64 \%, 99 \% \mathrm{CI}=-16.42,-3.61 \%$; $n=13)$. Thus, experience with a stimulus increases time spent in low-frequency oscillations and decreases time spent in high-frequency oscillations.

\section{Experience-dependent oscillations in V1 are stimulus specific}

We next sought to determine whether, like SRP, the shift in frequency composition of the LFP was stimulus specific. On day 7, in addition to the now highly familiar stimulus orientation, we presented a novel stimulus that was offset by $90^{\circ}$ from the familiar stimulus. Five blocks of each stimulus were pseudorandomly interleaved with each other. The familiar orientation induced a larger VEP than the novel orientation (Fig. 3A), as has been observed in numerous previous studies (Frenkel et al., 2006; Cooke et al., 2015; Fong et al., 2020). Statistics confirm that the familiar VEP is significantly larger than the novel VEP (Fig. 3B; 
A

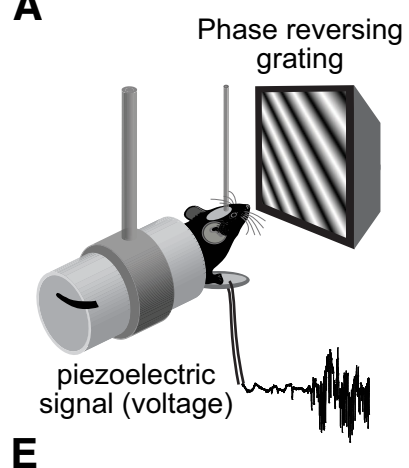

E

Example Example familiar frame novel frame

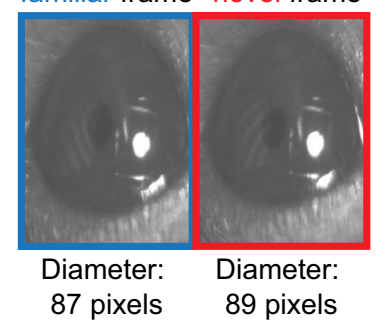

B

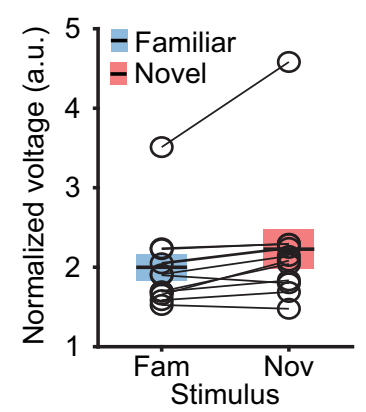

$\mathbf{F}$

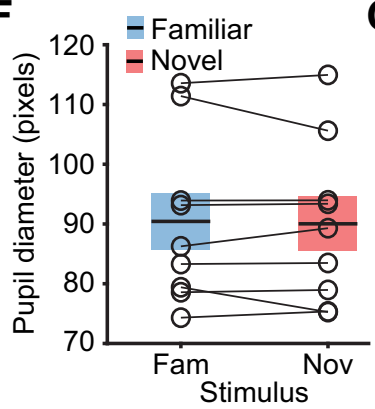

C

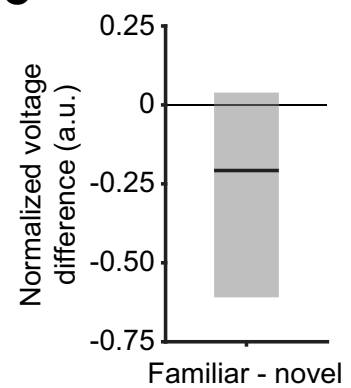

G

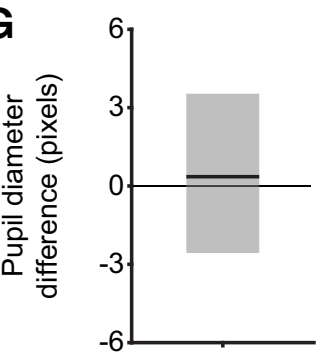

Familiar - novel
D

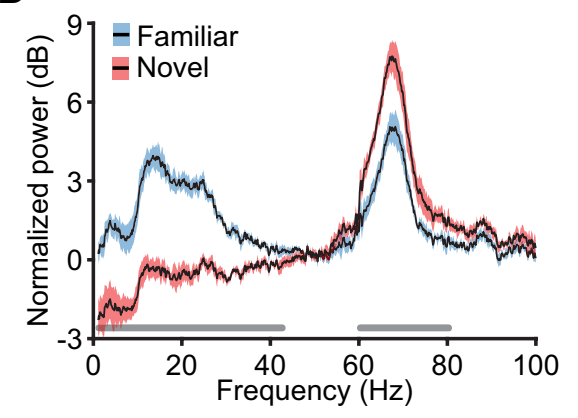

H

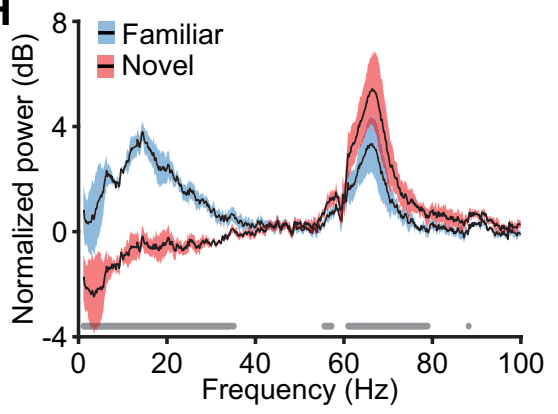

Familiar - novel

J

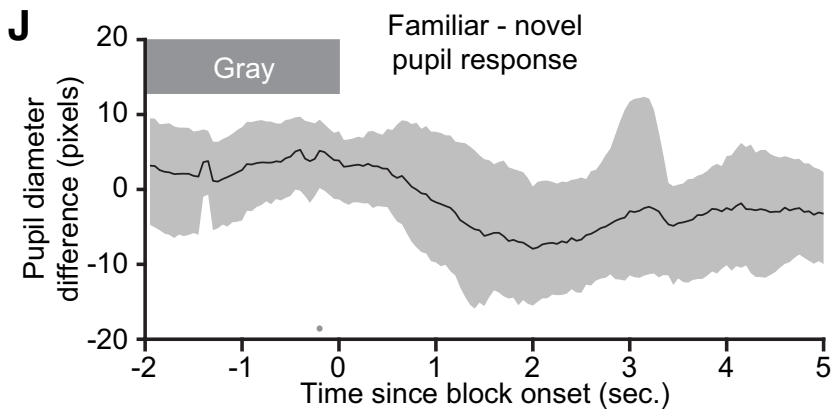

Figure 4. Movement and arousal do not account for the changes in layer 4 LFP frequency composition. $A$, We recorded forepaw movements of awake, head-fixed mice in response to phase-reversing sinusoidal grating stimuli. $\boldsymbol{B}$, The normalized piezo-electric voltage indicates there is no difference in the average forepaw movement during blocks of familiar (blue) and novel (red) stimuli over the time when LFPs were analyzed $(n=11)$. Averaged voltages are presented as mean \pm SEM. Open circles with connected lines show average normalized voltage from each animal. C, Nonparametric hierarchical bootstrapping results confirm that there is no piezo-electric voltage difference between familiar and novel stimuli. $\boldsymbol{D}$, In a subset (5) of these 11 mice, we simultaneously recorded the movement and LFP data. Presentation of a novel (red) stimulus increased high-frequency power and decreased low-frequency power compared with a familiar (blue) stimulus. Nonparametric hierarchical bootstrapping results confirm that the familiar spectrum is significantly different from the novel spectrum (marks near the $x$-axis indicate where the $99 \%$ confidence interval does not include zero). Averaged spectrums are presented as mean \pm SEM. E, Pupil size was measured as mice viewed blocks of familiar and novel stimuli. The familiar and novel frame from this exemplar mouse show the approximate population average size and location of the pupil. $\boldsymbol{F}$, The pupil diameter is similar as mice view familiar (blue) and novel (red) blocks of stimuli over the time when LFPs are analyzed $(n=9)$. Averaged pupil diameters are presented as mean \pm SEM. Open circles with connected lines show individual animal's average pupil diameter. $\mathbf{G}$, Nonparametric hierarchical bootstrapping results confirm that there is no pupil diameter difference between familiar and novel stimuli. $\boldsymbol{H}$, Same analysis of LFPs as in $\boldsymbol{D}$, but with a subset (4) of these nine mice in which we simultaneously recorded the pupillometry data and LFP data. $\boldsymbol{I}$, Pupil diameter at block onset $(n=9)$. Averaged pupil diameters are presented as mean \pm SEM. J, Nonparametric hierarchical bootstrapping results show that the familiar pupil diameter and novel pupil diameter are similar. Solid line represents the median value, and the shaded region reflects the $99 \%$ confidence interval. Marks near the $x$-axis indicate the $99 \%$ confidence interval does not include zero (thus the difference is statistically significant). The one statistically significant point in $\boldsymbol{J}$ is before the stimulus train appears and is likely a Type I error (false positive).

median peak-to-peak difference: $266.21 \mu \mathrm{V}, 99 \% \mathrm{CI}=262.03$, $270.00 \mu \mathrm{V} ; n=13)$. Consistent with our observation of changes in frequency composition with growing familiarity (Fig. 2), the familiar stimulus generated more low-frequency power and less high-frequency power in layer 4 LFP than the novel stimulus (Fig. 3C). Bootstrapping confirmed that the familiar orientation produced more low-frequency power (Fig. $3 D$; peak: $14.65 \mathrm{~Hz}$, median difference: $3.72 \mathrm{~dB}, 99 \% \mathrm{CI}=2.99,4.53 \mathrm{~dB} ; n=13$ ) and less high-frequency power than the novel orientation (Fig. 3D; peak: $67.63 \mathrm{~Hz}$, median difference: $-1.92 \mathrm{~dB}, 99 \% \mathrm{CI}=-2.80$, $-1.14 \mathrm{~dB} ; n=13)$. We observed similar results for the time spent in high- and low-frequency oscillations (Fig. $3 E$ ), with more time spent in low-frequency oscillations for the familiar orientation compared with the novel orientation (Fig. $3 F$; peak: $15.00 \mathrm{~Hz}$, median difference: $16.25 \%, 99 \% \mathrm{CI}=11.76,21.12 \% ; n=13)$ and less time spent in high-frequency oscillations (Fig. $3 F$; peak: $69.00 \mathrm{~Hz}$, median difference: $-11.83 \%, 99 \% \mathrm{CI}=-18.88$, $-6.32 \% ; n=13)$. Thus, oscillations within V1 are experience dependent and stimulus specific.

\section{Neither movement nor arousal account for the changes in layer 4 LFP frequency composition}

Studies have shown that locomotion can have a substantial effect on V1 oscillations and response properties in awake mice (Niell and Stryker, 2010; Bennett et al., 2013; Fu et al., 2014; Reimer et al., 2014; Vinck et al., 2015). Given the evidence that SRP and learned suppression of behavior both occur in tandem and require the same mechanisms (Cooke et al., 2015; Kaplan et al., 
2016), we were interested to understand whether oscillations that emerged in the LFP with growing stimulus familiarity were simply the result of reduced movement. Our previous analyses of behavior during SRP were restricted to the first few seconds after a transition from a gray screen to the stimulus, measuring an orienting or startle response that was more likely for novel than familiar stimuli (Cooke et al., 2015; Kaplan et al., 2016; Fong et al., 2020). The oscillations under investigation here extend throughout each stimulus block, over 200 s, so it was critical to analyze the animal's movement over this time period. To this end, we recorded piezoelectric activity that measured ongoing forepaw movement (Fig. 4A). The data shown in Figure 4 exclude the first $4 \mathrm{~s}$ of each block to remove the contribution of an orienting or startle response, but including these measurements in the average did not change the results. Analysis of the average forepaw movement, normalized to the gray screen (see above, Materials and Methods) revealed no difference if the mice viewed familiar or novel stimuli (Fig. $4 B ; n=11$ ). This was confirmed with nonparametric hierarchical bootstrapping (Fig. 4C; the confidence interval included zero; $n=11$ ). At no point within a phase reversal did novel stimuli elicit more forepaw movement than familiar stimuli or vice versa.

We simultaneously acquired the LFP and piezoelectric data in a subset of these animals over the same time interval. As expected from our previous results (Fig. 3), the familiar stimulus generated more low-frequency power (Fig. 4D; peak: $13.92 \mathrm{~Hz}$, median difference: $4.27 \mathrm{~dB}, 99 \% \mathrm{CI}=2.83,5.52 \mathrm{~dB} ; n$ $=5$ ) and less high-frequency power in the layer 4 LFP compared with the novel stimulus (Fig. 4D; peak: $67.63 \mathrm{~Hz}$, median difference: $-2.74 \mathrm{~dB}, 99 \% \mathrm{CI}=$ $-4.05,-1.56 \mathrm{~dB} ; n=5)$. Thus, the changes in spectral activity driven by stimulus novelty cannot simply be accounted for by movement.

Although movement itself may not account for the V1 oscillations that we have reported, changes in the LFP frequency composition could reflect global arousal shifts. Global arousal can be reliably monitored using pupillometry (Reimer et al., 2014, 2016). Thus, we also tracked pupil dilation as mice underwent the SRP paradigm (Fig. 4E). To remain consistent with the movement analysis, we excluded the first $4 \mathrm{~s}$

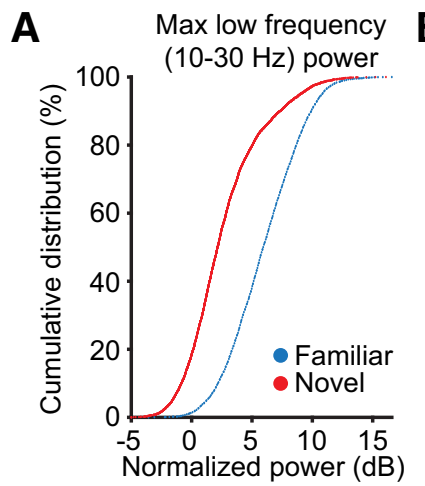

D

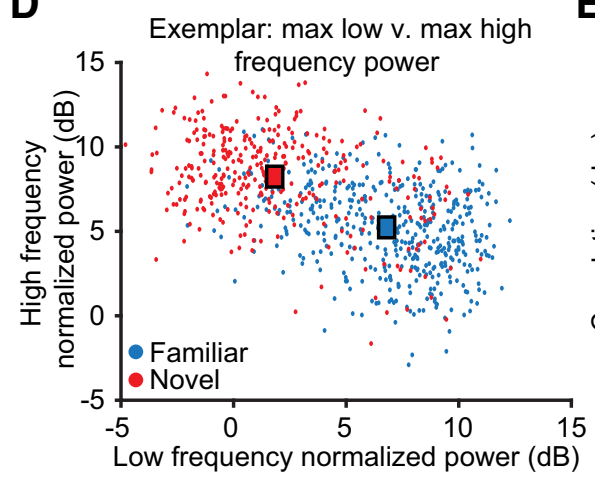

F
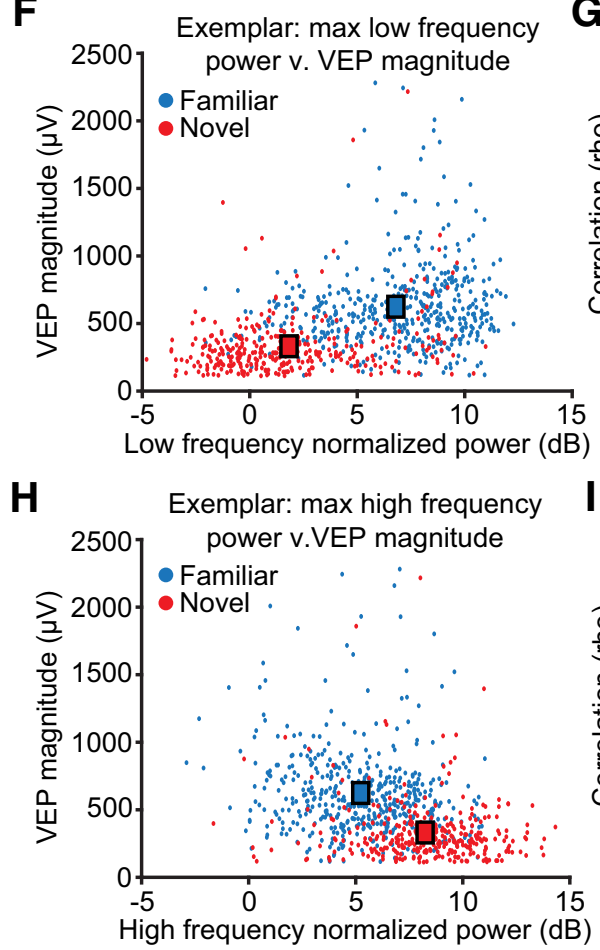

E
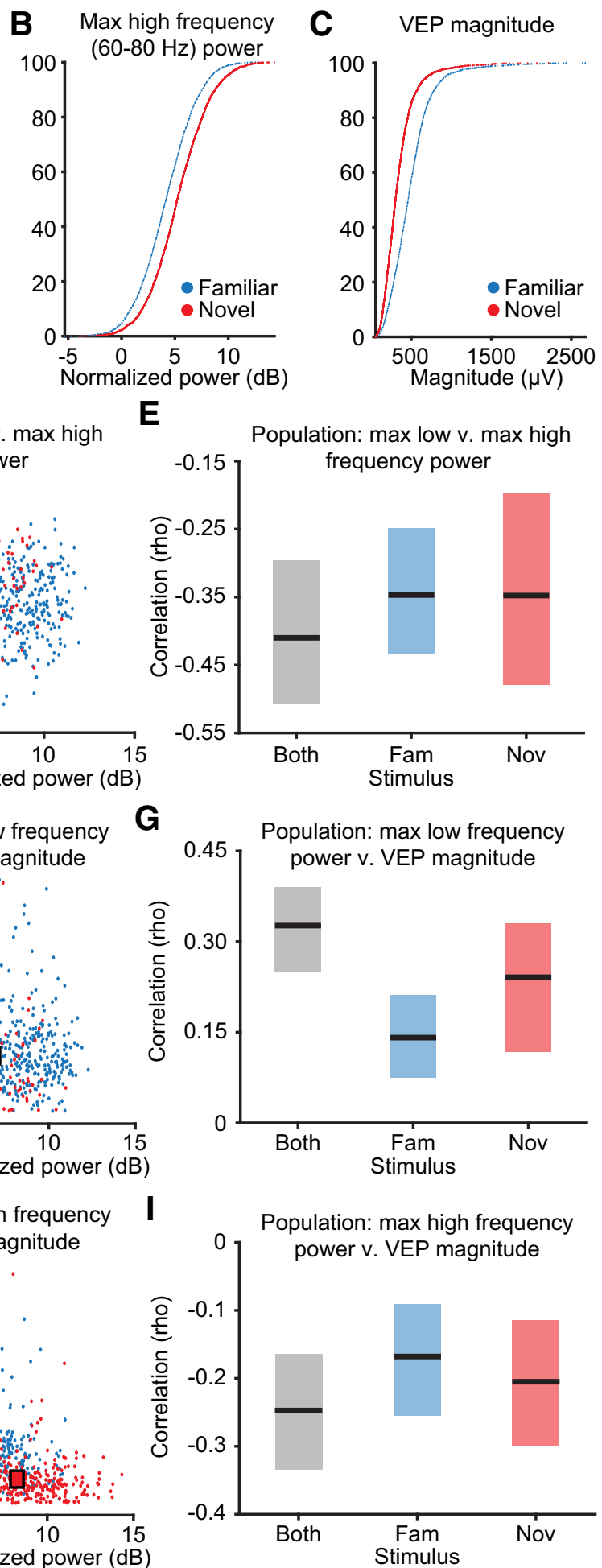

Figure 5. Oscillations and VEP magnitude correlate. $A$, LFPs and VEPs proximal to 500 stimulus phase reversals were studied in 13 mice. The maximum low-frequency $(10-30 \mathrm{~Hz})$ power is larger for familiar stimuli than novel stimuli. $\boldsymbol{B}$, The maximum high-frequency $(60-80 \mathrm{~Hz})$ power is larger for novel stimuli than familiar stimuli. C, The VEP magnitude is larger for familiar stimuli than novel stimuli. $\boldsymbol{D}$, An exemplar animal's scatter plot of each presentation's maximum low-frequency power and high-frequency power shows they are negatively correlated. $\boldsymbol{E}$, Nonparametric hierarchical bootstrapping results confirm that lowfrequency and high-frequency power are negatively correlated, regardless of stimulus. $\boldsymbol{F}$, Same as in $\boldsymbol{D}$, but for low-frequency power and VEP magnitude. $\boldsymbol{G}$, Same as in $\boldsymbol{E}$, but showing low-frequency power positively correlates with VEP magnitude. $\boldsymbol{H}$, Same as in $\boldsymbol{D}$, but for high-frequency power and VEP magnitude. I, Same as in $\boldsymbol{E}$, but showing high-frequency power negatively correlates with VEP magnitude. $\boldsymbol{E}, \mathbf{G}, \boldsymbol{I}$, Solid lines indicate the median value, and the shaded region reflects the $99 \%$ confidence interval. "Both" refers to a correlation analysis done with equal random sampling of both familiar and novel stimulus presentations. $\boldsymbol{D}, \boldsymbol{F}, \boldsymbol{H}$, Squares represent the midpoint of each group's scatter plot cloud. 

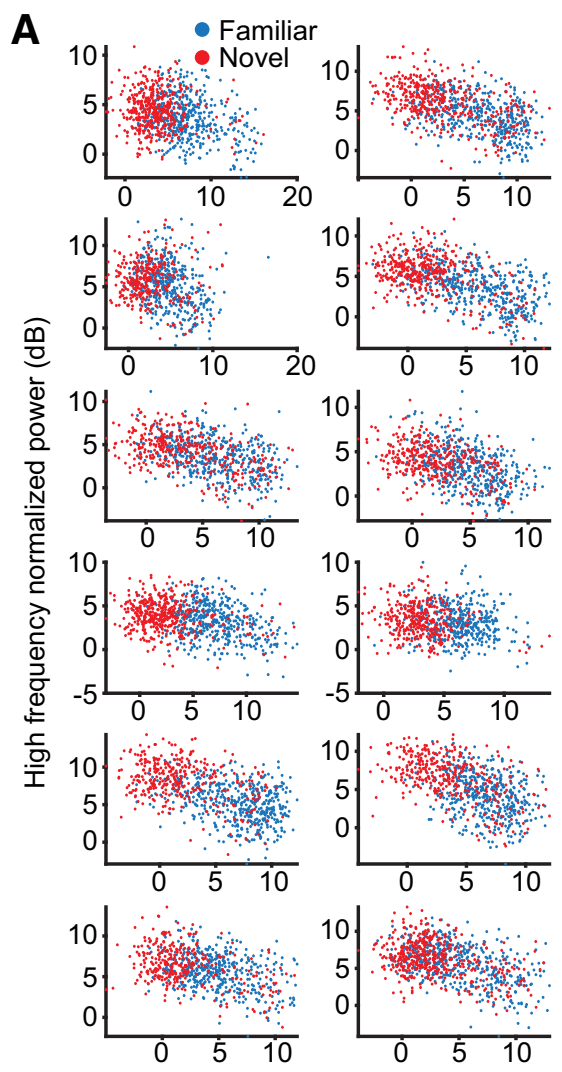

Low frequency normalized power $(\mathrm{dB})$
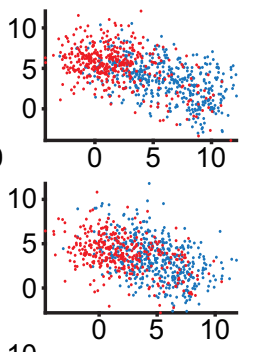

B
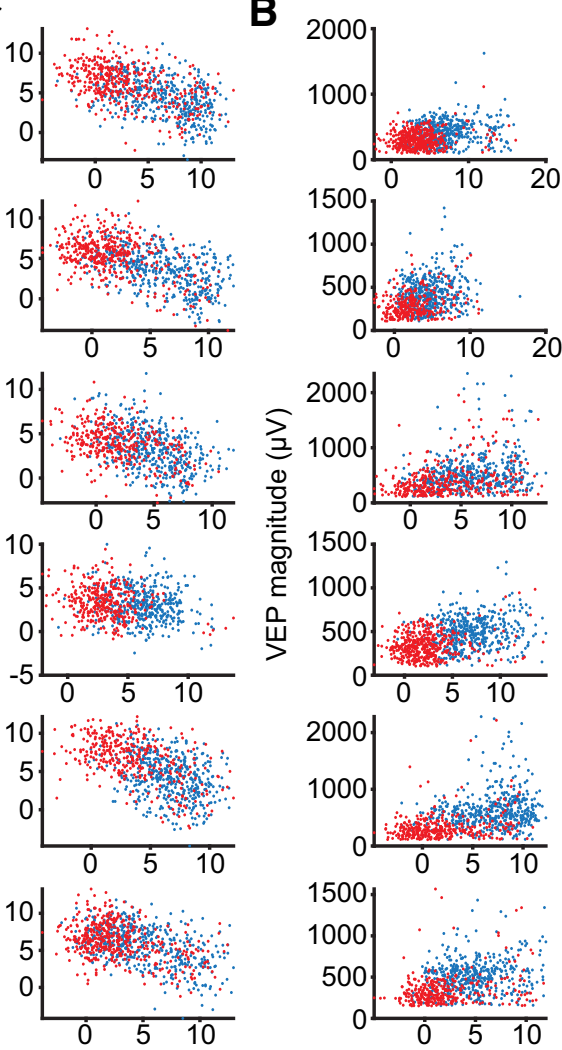
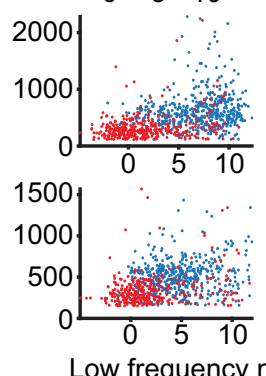
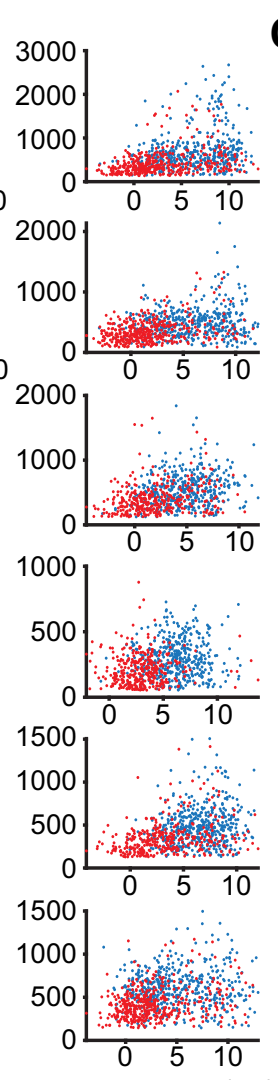

normalized power $(\mathrm{dB})$
C
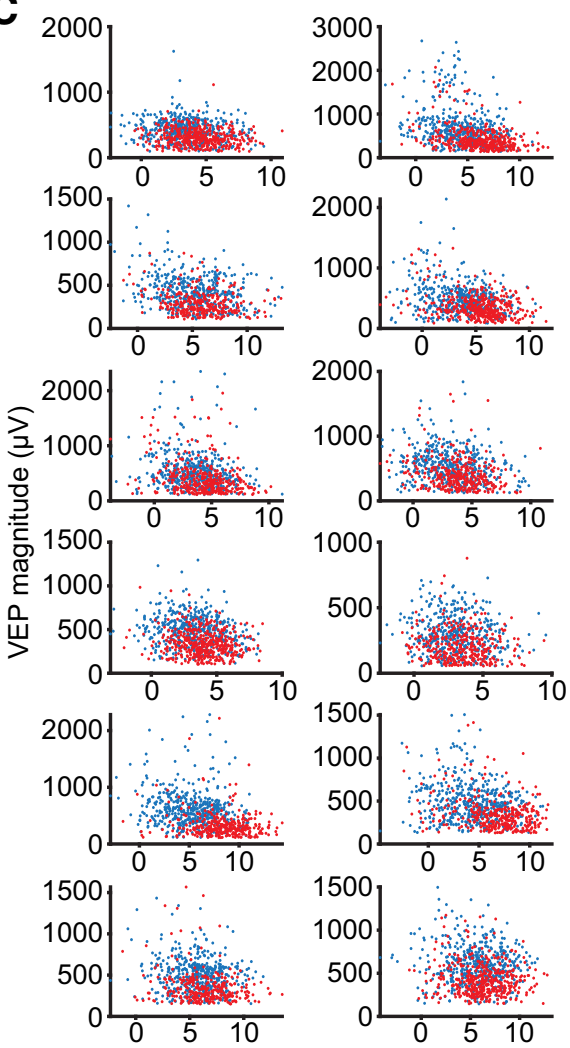

High frequency normalized power (dB)

Figure 6. Animal breakdown of correlation of VEP and oscillations. $\boldsymbol{A}$, Scatter plots of maximum low-frequency (10-30 Hz) power and maximum high-frequency (60-80 Hz) power for 12 of the 13 mice (the 13th is the exemplar in Fig. 5D). $\boldsymbol{B}$, Scatter plots of maximum low-frequency power and VEP magnitude for 12 of the 13 mice (the 13 th is the exemplar in Fig. $5 F$ ). $\boldsymbol{C}$, Scatter plots of maximum high-frequency power and VEP magnitude for 12 of the 13 mice (the 13th is the exemplar in Fig. 5H).

of each block of stimulation, but including them in the average did not change the results. As shown in Figure $4 F$, the average pupil diameter showed no observable or statistical difference between familiar and novel stimulus viewing conditions (Fig. $4 G$; the confidence interval included zero; $n=9)$. Additionally, at no point within a phase reversal did novel stimuli elicit a larger pupil diameter than familiar stimuli or vice versa, nor was there an appreciable difference in average pupil position $(\sim 4-6$ pixels).

We simultaneously acquired the LFP and pupillometry data over the same time interval in a subset of these animals. As expected from our previous results (Fig. 3), the familiar stimulus generated more low-frequency power (Fig. $4 \mathrm{H}$; peak: $14.04 \mathrm{~Hz}$, median difference: $3.99 \mathrm{~dB}, 99 \% \mathrm{CI}=3.01,5.09 \mathrm{~dB} ; n=4)$ and less high-frequency power in the layer 4 LFP compared with the novel stimulus (Fig. $4 \mathrm{H}$; peak: $67.02 \mathrm{~Hz}$, median difference: $-2.17 \mathrm{~dB}, 99 \% \mathrm{CI}=-3.18,-1.20 \mathrm{~dB} ; n=4)$. Thus, the familiarity-dependent changes in spectral activity that we have described cannot simply be accounted for by a global arousal shift.

Although there is no average pupil difference between familiar and novel stimuli (Fig. $4 F, G$ ), we were interested in whether there might be a difference at the start of a stimulus block. Our data show that novel stimuli cause a slightly elevated pupil diameter the few seconds after block onset compared with familiar stimuli (Fig. 4I; $n=9$ ). However, this difference is not statistically significant (Fig. 4J; all confidence intervals after block onset included zero; $n=9$ ). Given that the one significant point occurs before the stimulus starts, a Type 1 error (false positive) is likely. Thus, the pronounced familiarity-dependent spectral activity that we observe in our paradigm is unlikely to be accounted for by a temporary or sustained global arousal shift.

\section{Oscillations and VEP magnitudes correlate}

Given our previous measurements of increased VEP magnitude during SRP (Frenkel et al., 2006; Cooke and Bear, 2010; Cooke et al., 2015) and the concomitant changes in oscillations described here, we investigated the correlation between these two measures of experience-dependent plasticity. We analyzed the VEP magnitudes elicited by each phase reversal and the LFPs that immediately preceded them (see above, Materials and Methods). Changing the analysis window to the LFP after the VEP did not qualitatively change the results (data not shown). Cumulative distribution functions of all valid trials from all mice (see above, Materials and Methods) are shown for the maximum low-frequency $(10-30 \mathrm{~Hz})$ power, the maximum high-frequency (60$80 \mathrm{~Hz}$ ) power, and the VEP magnitude (Fig. $5 A-C$; $n=13$ mice). They show that familiar stimulus presentations have more lowfrequency power (Fig. 5A; two-sample Kolmogorov-Smirnov test, $p=0.00$ ), less high-frequency power (Fig. $5 B$; two-sample Kolmogorov-Smirnov test, $p=1.52 * 10^{-68}$ ), and larger VEP magnitudes (Fig. 5C; two-sample Kolmogorov-Smirnov test, $p=$ $2.89^{\star} 10^{-238}$ ) compared with novel stimulus presentations.

We then proceeded to correlate every combination of the three groups with each other. In Figure 5D,F,H we show the correlations from one representative animal whose dataset as a whole is most similar to that of the population average (all animals are shown in Fig. 6). In the exemplar, the maximum lowfrequency power negatively correlates with maximum high- 

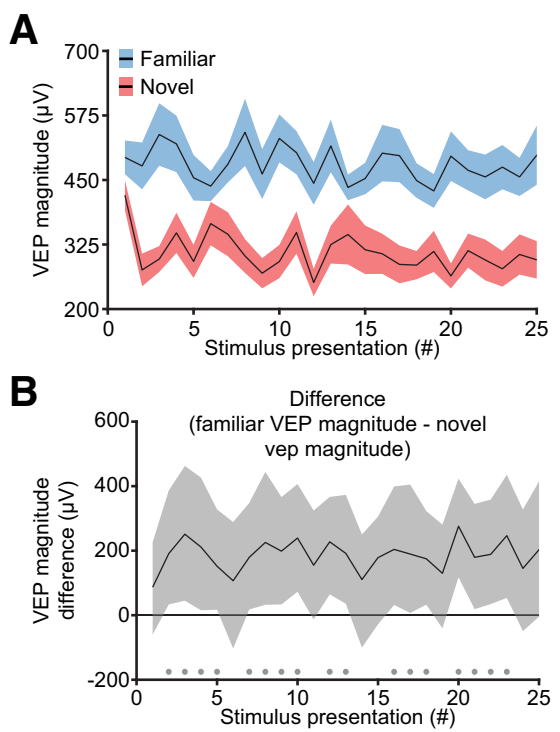

Figure 7. Differences in VEPs to familiar and novel stimuli emerge during a stimulus block. $\boldsymbol{A}$, In the 13 mice used for LFP analysis, we plot the change in VEP magnitude elicited by familiar and novel stimulus over the early trials from block onset. Presentation 1 corresponds to the VEP elicited by the transition from a gray screen to an oriented grating stimulus, and subsequent presentations correspond to phase reversals of this grating. Averaged VEP magnitude is presented as mean \pm SEM. $\boldsymbol{B}$, Nonparametric hierarchical bootstrapping results confirm previous findings (Kim et al., 2019) that VEP differences to familiar and novel stimuli only emerge after presentation 1. Marks near the $x$-axis indicate trials with a statistically significant difference.

frequency power (Fig. 5D). The population average for all animals shows a $\sim 40 \%$ negative correlation regardless of stimulus (Fig. $5 E$; median correlation for both: $-0.41,99 \% \mathrm{CI}=-0.51$, -0.30 ; median correlation for familiar: $-0.35,99 \% \mathrm{CI}=-0.43$ -0.25 ; median correlation for novel: $-0.35,99 \%$ CI $=-0.48$, $-0.20 ; n=13)$. There is no statistical difference between the correlation for familiar and novel stimuli (median correlation difference: $0.00,99 \% \mathrm{CI}=-0.14,0.15 ; n=13$; data not shown). In the exemplar, the maximum low-frequency power positively correlates with VEP magnitude (Fig. $5 F$ ). The population shows an $\sim 15-30 \%$ correlation depending on stimulus (Fig. $5 G$; median correlation for both: 0.33 , $99 \% \mathrm{CI}=0.25,0.39$; median correlation for familiar: $0.14,99 \% \mathrm{CI}=0.08,0.21$; median correlation for novel: $0.24,99 \%$ $\mathrm{CI}=0.12,0.33 ; n=13$ ). However, there is no statistical difference between the correlation for familiar and novel stimuli (median correlation difference: $-0.10,99 \% \mathrm{CI}=-0.21,0.03 ; n=13$, data not shown). Finally, in the exemplar, the maximum high-frequency power negatively correlates with VEP magnitude (Fig. $5 H$ ). The population shows an $\sim 20 \%$ negative correlation regardless of stimulus (Fig. 5I; median correlation for both: $-0.25,99 \% \mathrm{CI}=-0.33$, -0.16 ; median correlation for familiar: $-0.17,99 \% \mathrm{CI}=-0.25$, -0.09 ; median correlation for novel: -0.21 , 99\% CI $=-0.30$, $-0.11 ; n=13$ ). There is no statistical difference between the correlation for familiar and novel stimuli (median correlation difference: 0.04, 99\% CI $=-0.08,0.15 ; n=13$; data not shown). Thus, VEPs and low-frequency oscillatory power correlate regardless of stimulus novelty, and both grow with increased familiarity. These data are compatible with the hypothesis that the same underlying biology is responsible for both manifestations of SRP.

\section{Experience-dependent differences in V1 emerge after the first presentation}

A previous study showed that the initial VEPs and principal cell calcium transients elicited in layer 4 by the transition from a gray screen to an oriented grating stimulus are the same for both familiar and novel stimuli (Kim et al., 2019). The robust familiarnovel differences observed in time-averaged VEPs and cellular responses emerge during the course of a block of stimulation. Examination of VEPs in the same animals we have used for LFP analysis confirmed this prior finding (Fig. 7A). The first presentation of a stimulus after the gray period did not show a significant familiar-novel difference in VEP magnitude (Fig. $7 B$; trial 1 median peak-to-peak difference: $86.75 \mu \mathrm{V}, 99 \% \mathrm{CI}=-60.02$, $226.49 \mu \mathrm{V} ; n=13$ ). However, by the second presentation (corresponding to the first phase reversal), a familiar-novel difference was seen (Fig. $7 B$; trial 2 median peak-to-peak difference: $190.76 \mu \mathrm{V}, 99 \% \mathrm{CI}=33.33,386.49 \mu \mathrm{V} ; n=13)$. The emergence of SRP after the stimulus onset indicates recruitment of different circuits for familiar and novel stimuli (Kim et al., 2019).

These findings motivated us to compare the LFP oscillations proximal to the first and last stimulus presentations (both with the same $0^{\circ}$ phase, called a flip). Consistent with other measures of SRP, the first flip in a familiar block produced little low-frequency power but large high-frequency power, whereas the last flip displayed the expected increase in low-frequency power and decrease in high-frequency power (Fig. $8 A$ ). Compared with the first flip, the last flip in familiar blocks had more low-frequency power (Fig. $8 B$; peak: $21.48 \mathrm{~Hz}$, median difference: $3.16 \mathrm{~dB}, 99 \%$ $\mathrm{CI}=1.35,4.92 \mathrm{~dB} ; n=13$ ) and less high-frequency power (Fig. $8 B$; peak: $67.02 \mathrm{~Hz}$, median difference: $-2.72 \mathrm{~dB}, 99 \% \mathrm{CI}=$ $-4.49,-0.76 \mathrm{~dB} ; n=13)$. The change in high-frequency power, but not low-frequency power, was also seen for novel blocks (Fig. 8C). Compared with the first flip, the last flip in novel blocks had roughly the same low-frequency power (Fig. 8D; peak: $47.97 \mathrm{~Hz}$, median difference: $-1.80 \mathrm{~dB}, 99 \% \mathrm{CI}=-3.22$, $-0.35 \mathrm{~dB} ; n=13$ ) and less high-frequency power (Fig. $8 D$; peak: $68.73 \mathrm{~Hz}$, median difference: $-3.01 \mathrm{~dB}, 99 \% \mathrm{CI}=-4.92,-1.33$ $\mathrm{dB} ; n=13)$. Thus, prolonged exposure to a stimulus decreases high-frequency power regardless of stimulus familiarity, whereas only familiar stimuli show an increase in low-frequency power within a stimulus block.

We were next interested in exploring how quickly these modes of cortical activity emerge. At stimulus onset, the maximum normalized spectral power in the $10-30 \mathrm{~Hz}$ band quickly increased for familiar stimuli (Fig. 8E). Unexpectedly, power in this band also increased following novel stimulus onset, but the magnitude of the increase was less than that for a familiar stimulus (Fig. $8 F$ ). The maximum normalized spectral power in the $60-80 \mathrm{~Hz}$ band increased abruptly at the transition from black screen to gray screen, as expected (Saleem et al., 2017) and increased further on exposure to a novel stimulus (Fig. 8G). Power in this band decreased progressively over the first few phase reversals for both novel and familiar stimuli, but the familiar-novel difference was maintained (Fig. $8 H$ ).

We next assessed how the spectral power continues to evolve as visual stimulation continues. To do this, we first created a concatenated spectrum (Fig. 9A). Briefly, this concatenated spectrum is composed of the power spectrum for each phase reversal, excluding the time period containing the VEP (see above, Materials and Methods). In agreement with previous results (Fig. 8 ), the first few phase reversals showed a different oscillatory signature than the last few phase reversals. The concatenated spectrum appeared to be stable by the 25th phase reversal ( $50 \mathrm{~s}$ from stimulus onset). Thus, for each phase reversal, we extracted the maximum power in the $10-30 \mathrm{~Hz}$ and $60-80 \mathrm{~Hz}$ frequency bands, and used the average of this extracted maximum power for presentations $26-100$ as a comparator in our bootstrapping. 
A

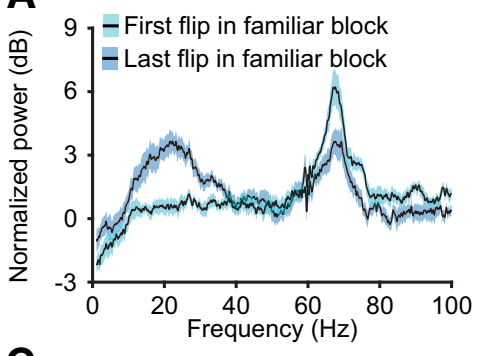

C
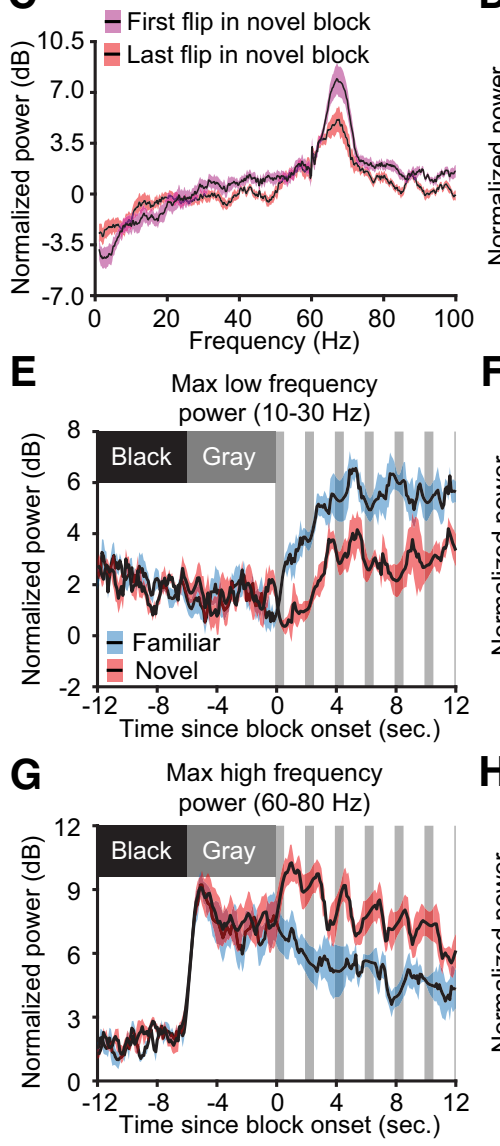

B

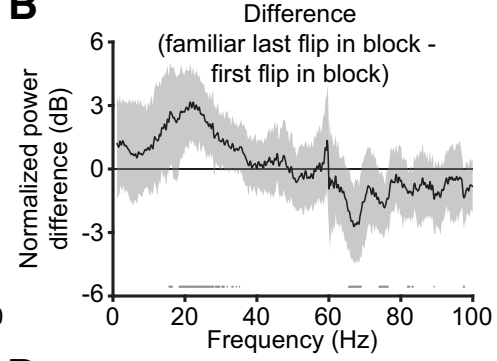

D

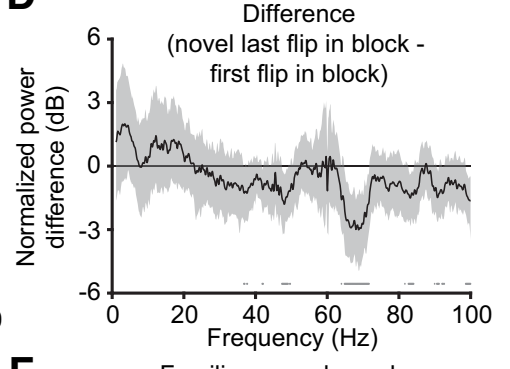

$\mathbf{F}$

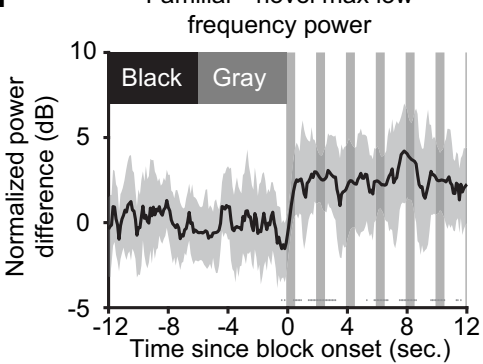

H

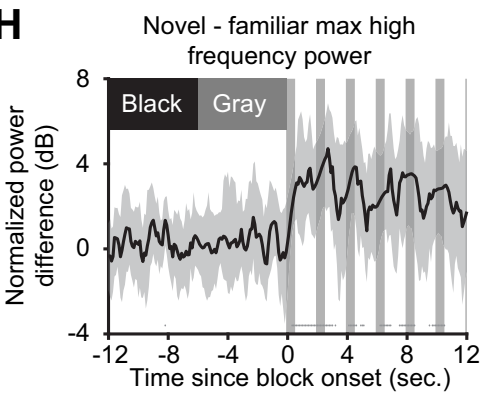

Figure 8. Experience-dependent oscillations in V1 change within a stimulus block. $\boldsymbol{A}$, The LFP following the onset of a familiar stimulus block (cyan) has increased high-frequency power and decreased low-frequency power compared with the last presentation of the same phase stimulus (blue). Averaged spectrums $(n=13)$ are presented as mean \pm SEM. $\boldsymbol{B}$, Nonparametric hierarchical bootstrapping results confirm that the last flip's spectrum is significantly different from the first flip's spectrum. Solid line represents the median value, and the shaded region reflects the $99 \%$ confidence interval. $\boldsymbol{C}$, The onset of a novel stimulus block (magenta) has increased high-frequency power compared with the last contrast reversal of the same phase (red). $\boldsymbol{D}$, Nonparametric hierarchical bootstrapping results for $\boldsymbol{C}$. $\boldsymbol{E}$, The maximum low-frequency power near block onset increases to a higher value for familiar stimuli compared with novel stimuli. Gray vertical bars represent periods where the LFP is contaminated by the VEP so interpolation was done to connect noncontaminated regions of the plot (see above, Materials and Methods). Averaged power is presented as mean \pm SEM. $\boldsymbol{F}$, Nonparametric hierarchical bootstrapping results confirm that familiar stimuli have more low-frequency power than novel. $\mathbf{G}$, Maximum high-frequency power near block onset increases to a larger value for a novel stimulus than a familiar one. $\boldsymbol{H}$, Same as in $\boldsymbol{F}$, but focused on high-frequency power at block onset. $\boldsymbol{B}, \boldsymbol{D}, \boldsymbol{F}, \boldsymbol{H}$, Marks near the $x$-axis indicate the $99 \%$ confidence interval does not include zero (thus the difference is statistically significant).

For familiar stimuli, low-frequency power started low then quickly increased to a steady value (Fig. 9B). Bootstrapping confirmed that only the first presentation is different from the last 75 trials (Fig. 9C; first presentation, median difference: $-2.54 \mathrm{~dB}$, $99 \% \mathrm{CI}=-3.71,-1.10 \mathrm{~dB} ; n=13$ ). For novel stimuli, there were interesting onset dynamics in the low-frequency band (Fig. 9D). Maximum power in the $10-30 \mathrm{~Hz}$ band started at the average level, increased transiently, then decayed back to average (Fig. $9 E$; fifth presentation, median difference: $1.95 \mathrm{~dB}, 99 \% \mathrm{CI}=0.76$, $3.11 \mathrm{~dB} ; n=13)$.

A similar analysis was conducted for high-frequency power. For familiar stimuli, power in the high-frequency band started high but quickly dropped to a steady level (Fig. 9F,G; first presentation, median difference: $2.03 \mathrm{~dB}, 99 \% \mathrm{CI}=0.95,3.03 \mathrm{~dB} ; n=$ 13). Similar kinetics were observed during novel stimulus viewing (Fig. 9H,I; first presentation, median difference: $2.16 \mathrm{~dB}, 99 \%$ $\mathrm{CI}=0.89,3.37 \mathrm{~dB} ; n=13$ ), but both the transient power and sustained power were shifted to greater values relative to familiar stimulus viewing.

\section{Layer $4 \mathrm{PV}+$ interneuron activity is suppressed as stimuli become familiar} Considerable evidence indicates that $\mathrm{PV}+$ inhibitory neurons play a critical role in the generation of cortical oscillations at frequencies $\geq 40 \mathrm{~Hz}$ (Cardin et al., 2009; Korotkova et al., 2010; Carlén et al., 2012; Gonzalez-Burgos and Lewis, 2012; Lewis et al., 2012; Kuki et al., 2015; Jadi et al., 2016; Polepalli et al., 2017; Veit et al., 2017). Given our observation here that novel stimuli elicit high-frequency oscillations, we performed experiments to measure the activity of layer $4 \mathrm{PV}+$ neurons over days during induction of SRP. We expressed GCaMP7 in genetically identified cortical neurons using a Cre-dependent conditional expression system and imaged cells with a 2-p microscope (Fig. $10 A, B)$. Only those cells that could be tracked across all days were included in the analysis. The average PV + cell activity decreased over days as the animal became familiar with the stimulus (Fig. 10C). Day 4 activity was significantly lower than day 1 activity (Fig. 10D; median difference: $-0.13 \mathrm{dF} / \mathrm{F}, 99 \% \mathrm{CI}=-0.22,-0.04 \mathrm{dF} / \mathrm{F}$; $n=9$ ), and less than gray-screen baseline activity. On day 5, when both familiar and novel stimuli were presented, the average $\mathrm{PV}+$ cell activity for each mouse showed a clear increase in activity during novel stimuli compared with familiar stimuli (Fig. 10E). Nonparametric hierarchical bootstrapping confirmed that familiar stimuli elicited less activity than novel stimuli (Fig. 10F; median difference: -0.14 $\mathrm{dF} / \mathrm{F}, 99 \% \mathrm{CI}=-0.18,-0.10 \mathrm{dF} / \mathrm{F} ; n=9)$. Thus, layer $4 \mathrm{PV}+$ cells in V1 are activated by novel stimuli and suppressed by familiar stimuli.

Layer 4 SOM + interneuron activity grows as stimuli become familiar

There is considerable evidence that $\mathrm{SOM}+$ inhibitory neurons contribute to low-frequency oscillations in the $15-30 \mathrm{~Hz}(\beta)$ 
A

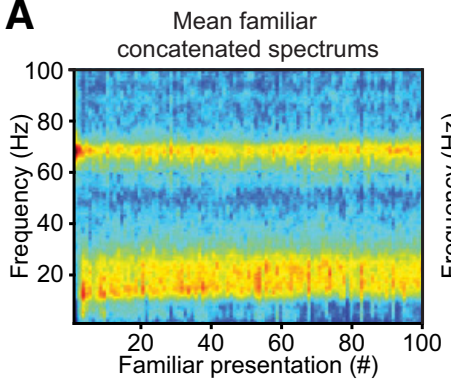

B
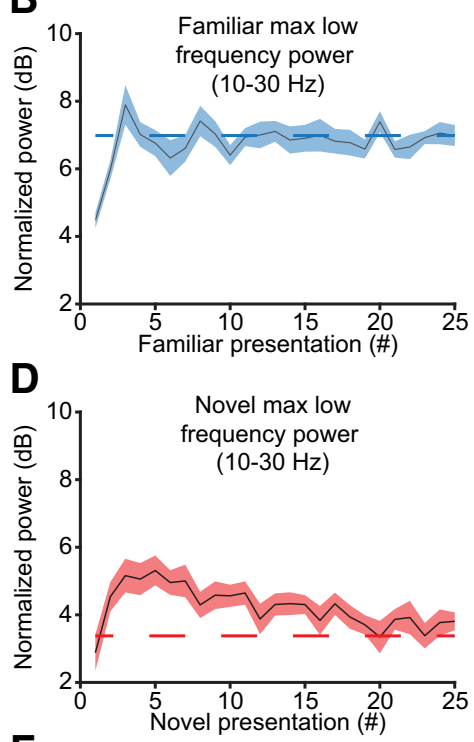

$\mathbf{F}$

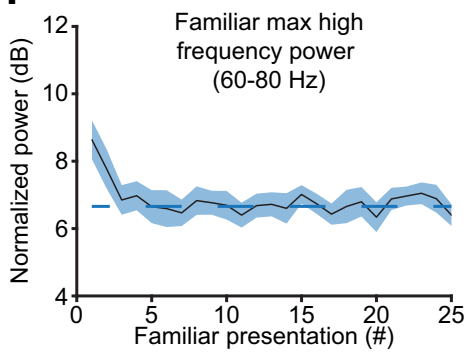

H

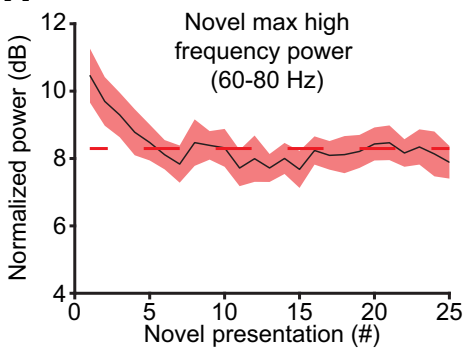

Mean novel

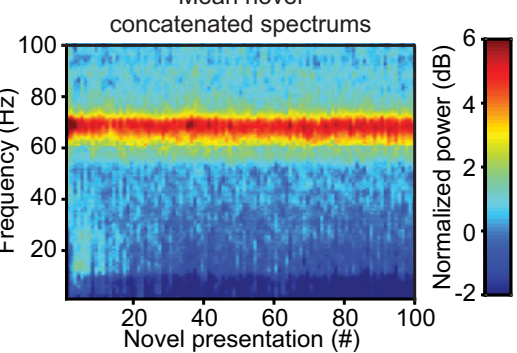

C

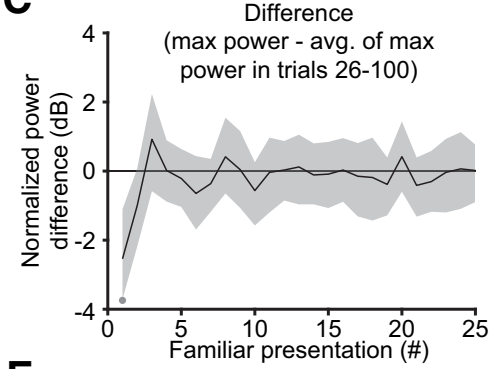

Difference

$\mathbf{E}$

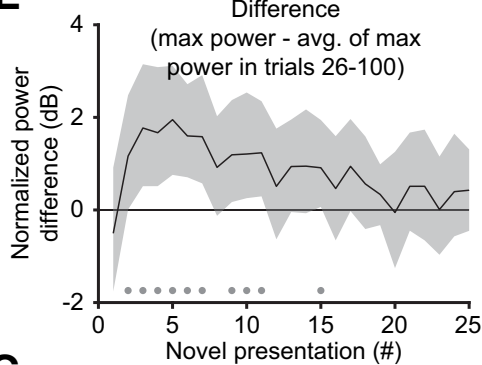

Difference

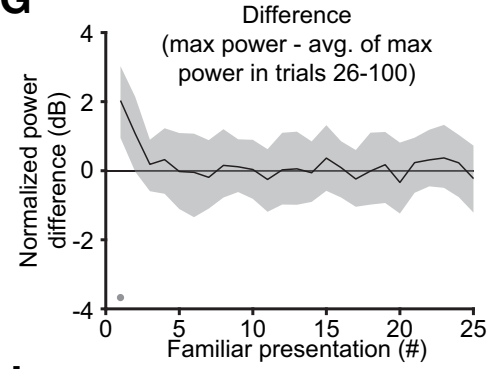

I

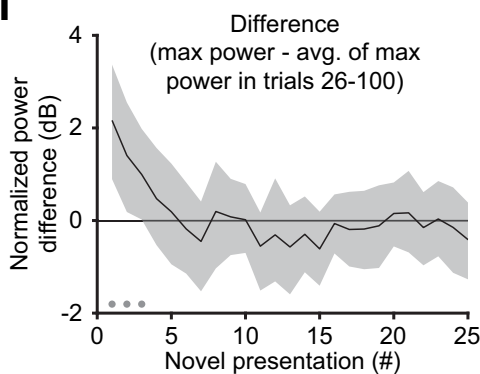

Figure 9. Emergence of experience-dependent oscillations in V1 during blocks of stimulation. $\boldsymbol{A}$, The average concatenated spectrum (see above, Materials and Methods) reveals short-term dynamics in oscillatory power for both familiar and novel stimuli $(n=13)$. B, For familiar stimuli, the maximum power in the $10-30 \mathrm{~Hz}$ band quickly increases to a steady value after the first presentation. $C$, Nonparametric hierarchical bootstrapping results confirm that only the first presentation shows significantly different changes from the average of the maximum power in the last 75 phase-reversals. $\boldsymbol{D}$, For novel stimuli, the maximum power in the $10-30 \mathrm{~Hz}$ band rises during the second through fifth presentations and slowly decays over the next 5-10 presentations. $\boldsymbol{E}$, Nonparametric hierarchical bootstrapping results confirm the significantly different spectral changes from the average of the maximum power in the last 75 phase reversals outlined in D. F, G, Same as $\boldsymbol{B}, \boldsymbol{C}$, but showing, for familiar stimuli, that the maximum power in the $60-80 \mathrm{~Hz}$ band quickly decreases to a steady-state value after the first presentation. $\boldsymbol{H}, \boldsymbol{I}$, Same as $\boldsymbol{D}, \boldsymbol{E}$, but showing, for novel stimuli, that the maximum power in the $60-80 \mathrm{~Hz}$ band decreases to a steady-state value after the first few presentations. Spectral power in $\boldsymbol{B}, \boldsymbol{D}, \boldsymbol{F}$, and $\boldsymbol{H}$ are presented as mean \pm SEM, and the dashed red line represents the average of the maximum power in the last 75 phase reversals. $C, E, G, I$, Marks near the $x$-axis indicate the $99 \%$ confidence interval does

range and become more active with experience (Kato et al., 2015; Makino and Komiyama, 2015; Hamm and Yuste, 2016; Chen et al., 2017; Veit et al., 2017; Khan et al., 2018). As we observed a sharp increase in oscillations at this frequency with increasing stimulus familiarity, we also measured the activity of layer $4 \mathrm{SOM}+$ neurons over days as SRP was induced. As with the PV+ cells, we expressed GCaMP7 in $\mathrm{SOM}+$ cortical neurons using a Cre-dependent conditional expression system and only analyzed cells that could be tracked across all days. The average SOM+ cell activity increased over days (Fig. 10G). Day 4 activity was significantly higher than day 1 activity (Fig. 10H; median difference: $0.22 \mathrm{dF} / \mathrm{F}, 99 \% \mathrm{CI}=0.07,0.38 \mathrm{dF} / \mathrm{F}$; $n=7$ ). On day $5, \mathrm{SOM}+$ cells were much more active during familiar stimulus viewing than during novel stimulus viewing (Fig. 10I). Bootstrapping confirms that familiar stimuli induced more activity than novel stimuli (Fig. 10J; median difference: $0.41 \mathrm{dF} / \mathrm{F}, 99 \% \mathrm{CI}=$ $0.28,0.56 \mathrm{dF} / \mathrm{F} ; n=7$ ).

Differences in the activity of PV+ and $\mathrm{SOM}+$ cells during familiar and novel stimulus viewing were robust and surprisingly uniform. In Figure 11 we compare activity for each neuron on days 1 and 4 as an initially novel stimulus becomes familiar and the activity to this now familiar stimulus to a novel orientation (Fig. $11 A, B ; n=1,251 \mathrm{PV}+$ neurons from nine mice; Fig. $11 C, D ; n=1,021$ $\mathrm{SOM}+$ neurons from seven mice). As this analysis shows, with very few exceptions, activity of PV + cells is higher to a novel stimulus than to a familiar stimulus. Conversely, virtually the entire network of SOM+ neurons in layer 4 is more active when a familiar stimulus is viewed than when a novel stimulus is presented.

Experience-dependent differences in PV+ and SOM + cell activity in V1 emerge over presentations

As with our study of the oscillatory power at block onset (Fig. 8), we sought to better understand how $\mathrm{PV}+$ and $\mathrm{SOM}+$ neurons in layer 4 change over the initial portion of visual stimulation. For both familiar and novel stimuli, PV + cell activity increased rapidly on the transition from gray to grating, and then diminished as the stimulus phase reversed (Fig. 12A). However the PV+ cell activity significantly differed between familiar and novel stimulus conditions less than a second from block onset (Fig. 12B; $0.70 \mathrm{~s}$,

not include zero (thus the difference is statistically significant). $\boldsymbol{C}, \boldsymbol{E}, \boldsymbol{G}, \boldsymbol{H}$, Additionally, the solid line represents the median value, and the shaded region reflects the $99 \%$ confidence interval. 
A

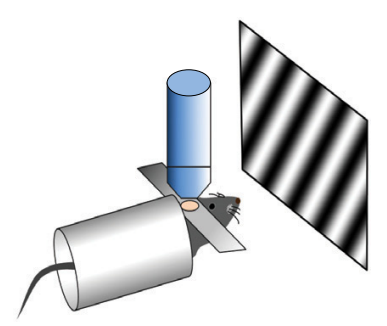

B

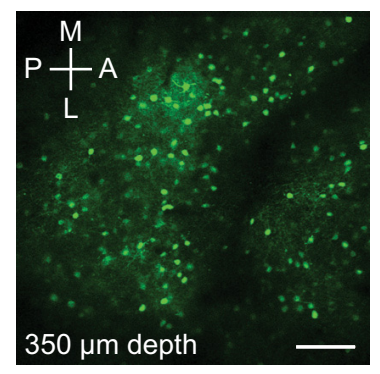

C

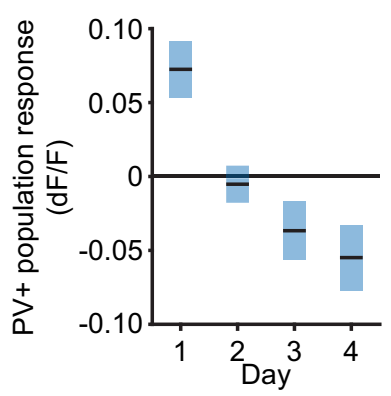

D

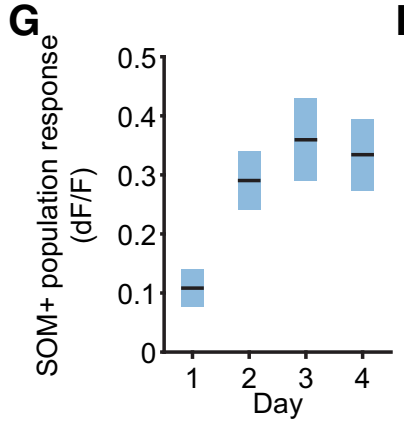

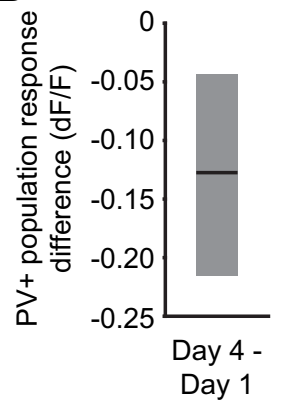

$\mathbf{E}$
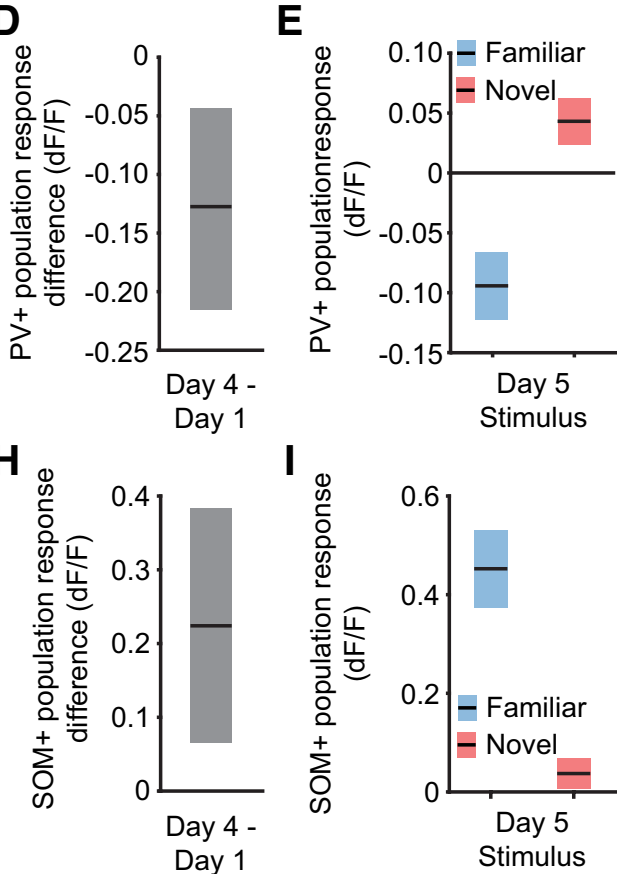

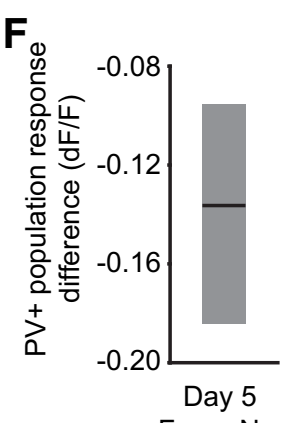

Fam - Nov

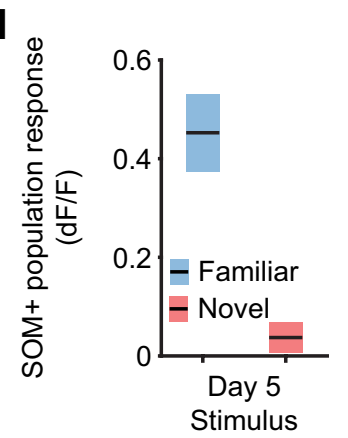

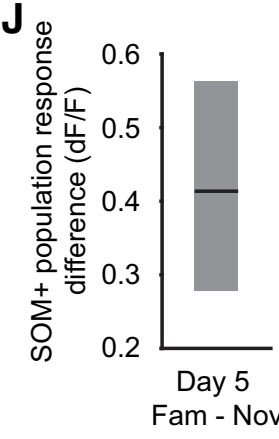

Figure 10. $\mathrm{PV}+$ and SOM + neurons in layer 4 respond differently to blocks of familiar and novel stimuli. $\boldsymbol{A}$, We recorded PV + cell activity or SOM + cell activity from layer 4 of $\mathrm{V} 1$ in awake, head-fixed mice in response to phase-reversing sinusoidal grating stimuli. $\boldsymbol{B}$, Image shows an example field of view. Scale bar, $100 \mu$ m. $\boldsymbol{C}$, PV+ cell activity gradually diminishes with stimulus experience (mean $\pm \mathrm{SEM} ; n=9$ ). D. Nonparametric hierarchical bootstrapping results confirm day 4 had less activity than day $1 . \boldsymbol{E}, 0 \mathrm{n}$ day 5 , PV + cell activity is elevated while the mouse views novel stimuli. $\boldsymbol{F}$, Nonparametric hierarchical bootstrapping results confirm familiar stimuli induce less PV+ cell activity than novel stimuli. G, SOM+ cell activity increases with stimulus experience (mean $\pm \mathrm{SEM} ; n=7$ ). $\boldsymbol{H}$, Nonparametric hierarchical bootstrapping results confirm day 4 had more activity than day $1 . \mathbf{I}$, On day 5 , SOM + cell activity is elevated while the mouse views familiar stimuli. $J$, Nonparametric hierarchical bootstrapping results confirm familiar stimuli induce more SOM+ cell activity than novel stimuli. All data are reported relative to the average interblock gray screen activity (see above, Materials and Methods). $C, E, G, I$, Activity is averaged across all cells for each animal and presented as the group mean \pm SEM. $\boldsymbol{D}, \boldsymbol{F}$, $\boldsymbol{H}, \boldsymbol{J}$, Solid black lines in indicate the median value for the difference, and the shaded regions reflect the $99 \%$ confidence interval.

median difference: $-0.082 \mathrm{dF} / \mathrm{F}, 99 \% \mathrm{CI}=-0.158,-0.012 \mathrm{dF} / \mathrm{F}$; $n=9)$.

$\mathrm{SOM}+$ cell activity also increased after the transition from gray screen to grating for both familiar and novel stimuli (Fig. $12 C)$. However, when the grating orientation was familiar, this increase occurred more rapidly than when the orientation was novel. The responses were clearly different after $8 \mathrm{~s}$ of stimulation (8.09 s, median difference: $0.117 \mathrm{dF} / \mathrm{F}, 99 \% \mathrm{CI}=0.002,0.334 \mathrm{dF} /$ $\mathrm{F} ; n=7)$, and first became statistically different within 4 seconds of block onset (Fig. 12D; $3.52 \mathrm{~s}$, median difference: $0.055 \mathrm{dF} / \mathrm{F}$, $99 \% \mathrm{CI}=0.001,0.128 \mathrm{dF} / \mathrm{F} ; n=7)$.

\section{Discussion}

A considerable body of evidence suggests that induction of SRP requires mechanisms that are shared with the phenomenon of LTP at excitatory synapses on principal neurons (Frenkel et al., 2006; Cooke and Bear, 2010; Aton et al., 2014; Cooke and Bear, 2014; Cooke et al., 2015). Here, we examined the hypothesis that expression of SRP depends on the differential recruitment of inhibitory networks by familiar and novel visual stimuli. Our results show that novel stimuli activate a population of $\mathrm{PV}+$ interneurons and elicit an increase in the power of high-frequency oscillations in the layer 4 LFP. Across days, as a stimulus becomes familiar, PV + cell activity and high-frequency oscillations subside, whereas SOM+ cell activity and low-frequency oscillations increase. Like other manifestations of SRP, these changes in LFP oscillations and interneuron activity are not subtle-they reflect dramatic shifts in the mode of visual information processing as a visual stimulus becomes familiar over days. Although the electrophysiological signature of stimulus recognition is not expressed immediately on the transition from a gray screen to a familiar stimulus (Kim et al., 2019), it does emerge quickly as evidenced by the rapid increase in low-frequency LFP power and VEP amplitude.

These observations inform and constrain the potential mechanisms that give rise to SRP. Although the current study was not designed to measure visual recognition behaviorally, extensive previous work has shown that SRP is a reliable biomarker of the changes in V1 that accompany formation, expression, and maintenance of visual recognition memory (Cooke et al., 2015; Kaplan et al., 2016; Fong et al., 2020). The changes reported by the LFP and VEPs occur over a time course that appears to be sufficiently fast to account for recognition measured behaviorally in this assay (Cooke et al., 2015).

Differential recruitment of mutually interacting networks of inhibitory neurons herald novelty detection and familiarity recognition

The first exposure of a mouse to an unexpected visual stimulus triggers a rapid increase in high-frequency LFP and PV + cell activity in layer 4 of V1 that continues throughout the entire block of stimulation. This is the dominant processing mode for novel visual stimuli in V1 of awake mice. One day later, when the stimulus is no longer novel, the $\mathrm{PV}+$ neurons cease to respond strongly. By the fourth day, presentation of the now familiar stimulus instead causes suppression of a substantial fraction of $\mathrm{PV}+$ neurons in layer 4, and, unsurprisingly, there is a clear decrease in the power and duration of high-frequency oscillations of the LFP. Over the same time course, there is a substantial increase in the magnitude of the VEP elicited by the familiar 
stimulus. These observations are consistent with previous findings that silencing of $\mathrm{PV}+$ neurons locally within $\mathrm{V} 1$ causes a decrease in $60-80 \mathrm{~Hz}$ LFP power (Chen et al., 2017; Veit et al., 2017) and an increase in VEPs that mimics and occludes SRP (Kaplan et al., 2016). Conversely, it has been shown that optogenetic stimulation of the PV + neurons reverses SRP expression in the VEP (Kaplan et al., 2016). Together, these observational and interventional data suggest that expression of SRP in the VEP may be accounted for entirely by differential recruitment of $\mathrm{PV}+$ interneurons by familiar and novel visual stimuli.

In layer 4 of the sensory cortex, $\mathrm{PV}+$ inhibitory neurons are known to be strongly inhibited by SOM+ neurons (Pfeffer et al., 2013; Xu et al., 2013). Inspired by a study in the auditory cortex showing that passive sound exposure upregulates SOM+ neuron activity in layer 3 (Kato et al., 2015), we examined the effect of visual grating familiarity on the activity of SOM+ neurons in layer 4 of $\mathrm{V} 1$. The data show a robust and strikingly uniform increase in the activity of SOM+ cells as the stimulus becomes familiar. As expected from previous work (Chen et al., 2017; Veit et al., 2017), engagement of SOM + cells by the familiar stimulus was associated with an increase in the power and duration of lowfrequency LFP oscillations. This is the dominant processing mode for familiar stimuli in V1 of awake mice.

It has been shown by others that the activity of SOM+ and $\mathrm{PV}+$ neurons of the mouse V1 is strongly modulated by locomotion (Fu et al., 2014). Our previous studies have shown that reflexive forepaw movements (vidgets) occur for the first few seconds following the transition from a gray screen to a novel grating and that this response diminishes over days as the grating becomes familiar (Cooke et al., 2015; Kaplan et al., 2016; Fong et al., 2020). However, using this same approach to monitor continuous forepaw movement over the entire 3.5 min block of phasereversing stimuli, we observed no familiarnovel differences. This finding suggests that movement is not a confounding variable for the interpretation of our LFP or imaging data collected over the same time period. Moreover, both populations of interneurons in layer 4 show comparable increases when movement occurs during visual stimulation (Pakan et al., 2016). Thus, the differential recruitment of these inhibitory networks by familiar and novel stimuli is unlikely to be accounted for by movement.

Cortical responsiveness and oscillations are influenced by transitions in global
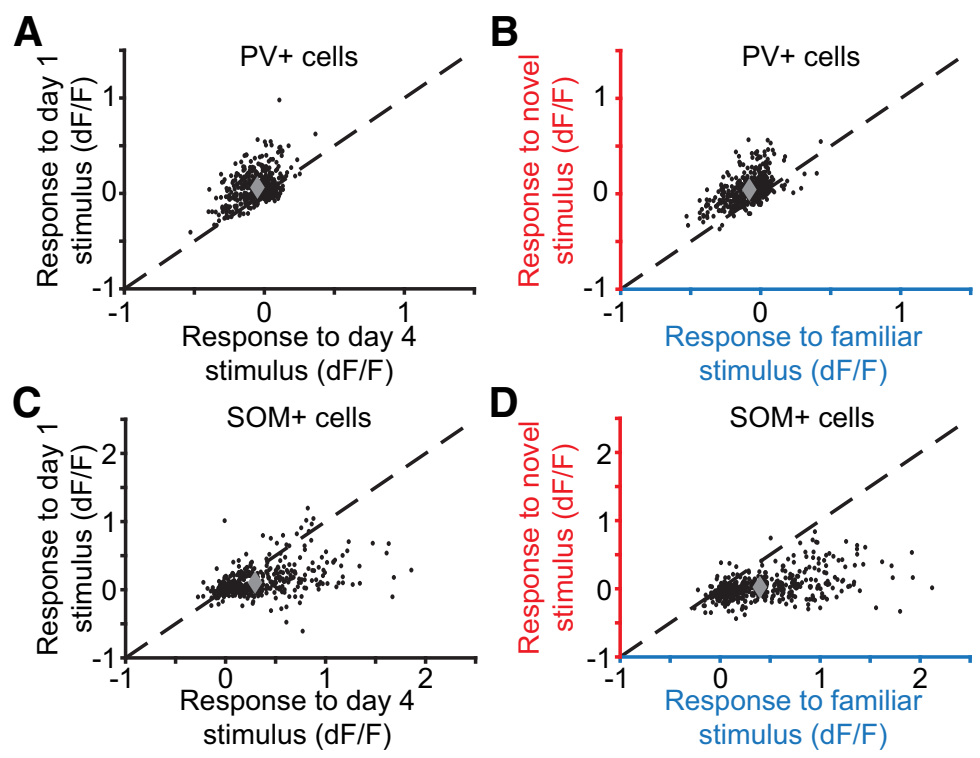

Figure 11. Familiar-novel differences in activity of parvalbumin-expressing and somatostatin-expressing neurons are remarkably uniform. $\boldsymbol{A}$, Plotted are the responses of each $\mathrm{PV}+$ neuron recorded during visual stimulation with a phasereversing grating of the same orientation on the first and fourth day ( $n=1,251 \mathrm{PV}+$ neurons from 9 mice). $\boldsymbol{B}$, Responses of the same PV+ neurons on day 5, comparing the familiar and novel stimulus orientations. $C$, Responses of each SOM+ cell plotted on day 1 versus day 4 of viewing the same oriented stimulus ( $n=1,021 \mathrm{SOM}+$ neurons from 7 mice). $\boldsymbol{D}$, Responses of the same SOM+ neurons on day 5, comparing the familiar and novel stimulus orientations. All data reported relative to the average interblock gray screen activity (see above, Materials and Methods). $\boldsymbol{A}-\boldsymbol{D}$, Dashed line is the identity line $y=x$.
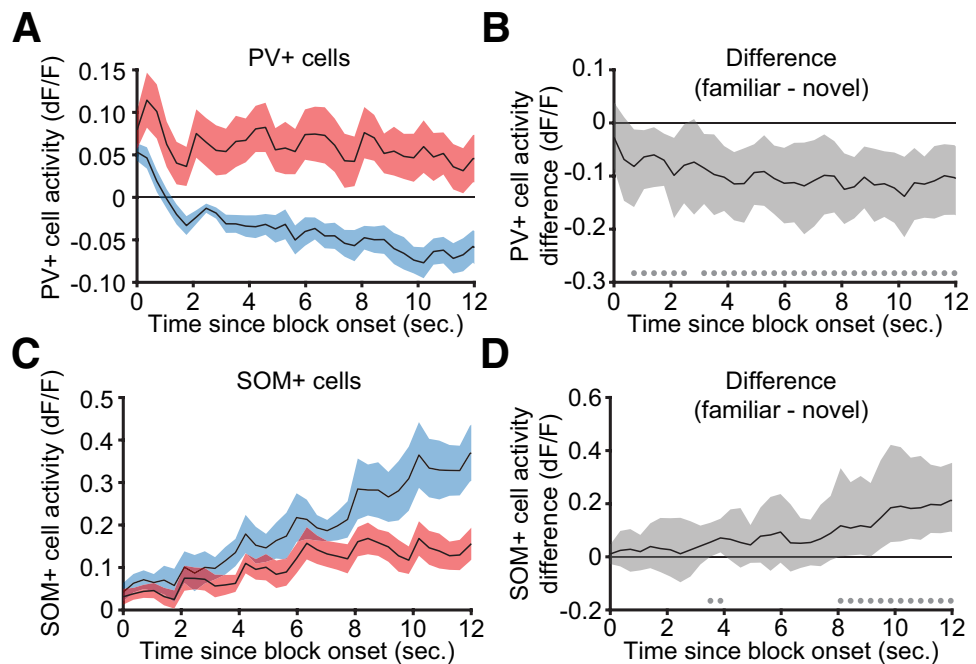

Figure 12. Experience-dependent differences in the activity of PV + and $\mathrm{SOM}+$ emerge during blocks of stimulation. $\boldsymbol{A}, \mathrm{PV}+$ cell activity from layer 4 of $\mathrm{V} 1$ in nine awake, head-fixed mice in response to the transition from gray screen (time 0 ) and during subsequent phase-reversals of sinusoidal grating stimuli every $2 \mathrm{~s}$. Both familiar (blue) and novel (red) showed an increase in PV+ cell activity at block onset followed by a decrease that was more pronounced during familiar stimulus viewing. $\boldsymbol{B}$, Nonparametric hierarchical bootstrapping results confirm that PV + cell activity during novel blocks is larger than familiar blocks as early as $1 \mathrm{~s}$ after stimulus onset. $\boldsymbol{C}, \boldsymbol{D}$, Same as in $\boldsymbol{A}, \boldsymbol{B}$, but measuring SOM + cell activity in seven mice. SOM + cell activity is increased after block onset during stimulation with both familiar (blue) and novel (red) gratings. However, the increase during novel stimulus viewing is less than during familiar stimulus viewing. Nonparametric hierarchical bootstrapping results confirm that $\mathrm{SOM}+$ cell activity during familiar blocks is larger than novel blocks as early as 3-4 s. A, C, Activity is averaged across all cells for each animal and presented as the group mean \pm SEM. $\boldsymbol{B}, \boldsymbol{D}$, Solid black lines indicate the median value for the difference, and the shaded regions reflect the $99 \%$ confidence interval. $\boldsymbol{B}, \boldsymbol{D}$, Marks near the $x$-axis indicate the $99 \%$ confidence interval does not include zero (thus the difference is statistically significant) 
brain states, mediated by diffusely projecting neuromodulatory systems (Hasenstaub et al., 2007; Bennett et al., 2013; Luczak et al., 2013; Kissinger et al., 2018). Our findings could be explained if novel stimuli produce more sustained arousal than familiar stimuli. However, we monitored global arousal through pupillometry (Reimer et al., 2014; Vinck et al., 2015) and found no differences in pupil size during familiar or novel stimulus viewing. Furthermore, an expression mechanism based on slowly conducting modulatory systems seems unlikely considering (1) the speed of the transition in the LFP that heralds familiarity recognition and (2) the fact that the essential synaptic modifications underlying SRP reside within V1.

\section{Putting the pieces together}

The current study adds important new pieces to the puzzle of SRP and, by extension, visual recognition memory in V1. The original description of SRP was the robust increase in the magnitude of the VEP elicited by phase reversing a familiar stimulus (Frenkel et al., 2006), reflecting a net increase in positive current flowing into (most likely) radially oriented apical dendrites (Cooke et al., 2015). Combined with our previous findings (Kaplan et al., 2016), the current results indicate that the simplest explanation for this increase in net current flow is reduced PV+ mediated inhibition. Given the known connectivity of SOM+ cells and their involvement in the generation of $10-30 \mathrm{~Hz}(\alpha / \beta)$ oscillations in the LFP (Kato et al., 2015; Makino and Komiyama, 2015; Hamm and Yuste, 2016; Veit et al., 2017), an appealing hypothesis is that the experience-dependent increase in the activation of SOM+ neurons by familiar stimuli accounts for suppression of PV + neurons and potentiation of VEPs. This simple model is challenged somewhat by our imaging experiments suggesting that the activity of the entire population of $\mathrm{SOM}+$ cells in layer 4 is relatively slow to discriminate familiar and novel stimuli following block onset (Fig. 12C,D). However, it may be that only a threshold number of SOM+ neurons needs to be recruited to suppress the $\mathrm{PV}+$ neurons at the earliest time points. In addition, the differential response kinetics of SOM+ cells may be underestimated as a consequence of the poor temporal resolution of calcium imaging methods. Indeed, if 10$30 \mathrm{~Hz}$ oscillations report recruitment of SOM+ cells in V1, then the activity of these neurons increases amply fast to account for VEP potentiation as soon as it can be detected (Fig. 8E,F). Regardless, testing this model will require direct manipulation of $\mathrm{SOM}+$ cell activity in future studies.

A full description of SRP must also account for the additional observations that when measured with calcium imaging, layer 4 principal cell activity is reduced by familiarity (Kim et al., 2019). These calcium signals reflect changes in sustained activity as they do not report augmented peak firing rates that occur with each familiar phase reversal (Aton et al., 2014; Cooke et al., 2015; Clawson et al., 2018), but they do mirror the habituation of behavioral responses (Cooke and Bear, 2015). It is tempting to speculate that recruitment of SOM + neurons by familiar stimuli could be responsible for multiple facets of SRP-suppression of both principal cell and PV + activity in layer 4 , as well as the behavioral response. We are still left with the question of how $\mathrm{SOM}+$ neurons become more active as a stimulus is learned. There are many possibilities that remain to be explored, but available data indicate there is an essential role for mechanisms of excitatory synaptic plasticity (Cooke and Bear, 2010, 2014).

The data suggest that the PV + neurons, which are known to receive a more powerful thalamic input than glutamatergic principal neurons (Cruikshank et al., 2007), are highly engaged by unexpected feedforward sensory input, whereas SOM+ neurons are recruited by a recognition memory trace within V1 (Frenkel et al., 2006; Cooke and Bear, 2010; Cooke et al., 2015). Thus, the novelty response may reflect a default feedforward, plasticitypromoting state that persists until a stimulus is recognized as familiar. This putative organization is similar conceptually to the comparator model of habituation (Sokolov, 1963) in which sensory input is constantly compared with engrams distributed throughout the cortex, and only when a match occurs is inhibition recruited to suppress reflexive behavioral output. Future studies aimed at dissecting the interplay between these two inhibitory neuronal populations within the framework of comparator/adaptive filtration systems will be of great interest.

\section{References}

Aton SJ, Suresh A, Broussard C, Frank MG (2014) Sleep promotes cortical response potentiation following visual experience. Sleep 37:1163-1170.

Bennett C, Arroyo S, Hestrin S (2013) Subthreshold mechanisms underlying state-dependent modulation of visual responses. Neuron 80:350-357.

Bokil H, Andrews P, Kulkarni JE, Mehta S, Mitra PP (2010) Chronux: a platform for analyzing neural signals. J Neurosci Methods 192:146-151.

Caplan JB, Madsen JR, Raghavachari S, Kahana MJ (2001) Distinct patterns of brain oscillations underlie two basic parameters of human maze learning. J Neurophysiol 86:368-380.

Cardin JA, Carlén M, Meletis K, Knoblich U, Zhang F, Deisseroth K, Tsai LH, Moore CI (2009) Driving fast-spiking cells induces gamma rhythm and controls sensory responses. Nature 459:663-667.

Carlén M, Meletis K, Siegle JH, Cardin JA, Futai K, Vierling-Claassen D, Rühlmann C, Jones SR, Deisseroth K, Sheng M, Moore CI, Tsai LH (2012) A critical role for NMDA receptors in parvalbumin interneurons for gamma rhythm induction and behavior. Mol Psychiatry 17:537-548.

Chalk M, Herrero JL, Gieselmann MA, Delicato LS, Gotthardt S, Thiele A (2010) Attention reduces stimulus-driven gamma frequency oscillations and spike field coherence in V1. Neuron 66:114-125.

Chen G, Zhang Y, Li X, Zhao X, Ye Q, Lin Y, Tao HW, Rasch MJ, Zhang X (2017) Distinct inhibitory circuits orchestrate cortical beta and gamma band oscillations. Neuron 96:1403-1418.e6.

Chen TW, Wardill TJ, Sun Y, Pulver SR, Renninger SL, Baohan A, Schreiter ER, Kerr RA, Orger MB, Jayaraman V, Looger LL, Svoboda K, Kim DS (2013) Ultrasensitive fluorescent proteins for imaging neuronal activity. Nature 499:295-300.

Clawson BC, Durkin J, Suresh AK, Pickup EJ, Broussard CG, Aton SJ (2018) Sleep promotes, and sleep loss inhibits, selective changes in firing rate, response properties and functional connectivity of primary visual cortex neurons. Front Syst Neurosci 12:40.

Cooke SF, Bear MF (2010) Visual experience induces long-term potentiation in the primary visual cortex. J Neurosci 30:16304-16313.

Cooke SF, Bear MF (2014) How the mechanisms of long-term synaptic potentiation and depression serve experience-dependent plasticity in primary visual cortex. Philos Trans R Soc Lond B Biol Sci 369:20130284.

Cooke SF, Bear MF (2015) Visual recognition memory: a view from V1. Curr Opin Neurobiol 35:57-65.

Cooke SF, Komorowski RW, Kaplan ES, Gavornik JP, Bear MF (2015) Visual recognition memory, manifested as long-term habituation, requires synaptic plasticity in V1. Nat Neurosci 18:262-271.

Cruikshank SJ, Lewis TJ, Connors BW (2007) Synaptic basis for intense thalamocortical activation of feedforward inhibitory cells in neocortex. Nat Neurosci 10:462-468.

Fong MF, Finnie PS, Kim T, Thomazeau A, Kaplan ES, Cooke SF, Bear MF (2020) Distinct laminar requirements for NMDA receptors in experience-dependent visual cortical plasticity. Cereb Cortex 30:2555-2572.

Frenkel MY, Sawtell NB, Diogo AC, Yoon B, Neve RL, Bear MF (2006) Instructive effect of visual experience in mouse visual cortex. Neuron 51:339-349.

Fu Y, Tucciarone JM, Espinosa JS, Sheng N, Darcy DP, Nicoll RA, Huang ZJ, Stryker MP (2014) A cortical circuit for gain control by behavioral state. Cell 156:1139-1152.

Gonzalez-Burgos G, Lewis DA (2012) NMDA receptor hypofunction, parvalbumin-positive neurons, and cortical gamma oscillations in schizophrenia. Schizophr Bull 38:950-957. 
Hamm JP, Yuste R (2016) Somatostatin interneurons control a key component of mismatch negativity in mouse visual cortex. Cell Rep 16:597-604.

Hasenstaub A, Sachdev RN, McCormick DA (2007) State changes rapidly modulate cortical neuronal responsiveness. J Neurosci 27:9607-9622.

Jadi MP, Behrens MM, Sejnowski TJ (2016) Abnormal gamma oscillations in $\mathrm{N}$-methyl-D-aspartate receptor hypofunction models of schizophrenia. Biol Psychiatry 79:716-726.

Kaneko M, Stryker MP (2014) Sensory experience during locomotion promotes recovery of function in adult visual cortex. Elife 3:e02798.

Kaneko M, Fu Y, Stryker MP (2017) Locomotion induces stimulus-specific response enhancement in adult visual cortex. J Neurosci 37:3532-3543.

Kaplan ES, Cooke SF, Komorowski RW, Chubykin AA, Thomazeau A, Khibnik LA, Gavornik JP, Bear MF (2016) Contrasting roles for parvalbumin-expressing inhibitory neurons in two forms of adult visual cortical plasticity. Elife 5:e11450.

Kato HK, Gillet SN, Isaacson JS (2015) Flexible sensory representations in auditory cortex driven by behavioral relevance. Neuron 88:1027-1039.

Khan AG, Poort J, Chadwick A, Blot A, Sahani M, Mrsic-Flogel TD, Hofer SB (2018) Distinct learning-induced changes in stimulus selectivity and interactions of GABAergic interneuron classes in visual cortex. Nat Neurosci 21:851-859.

Kim T, Chaloner FA, Cooke SF, Harnett MT, Bear MF (2019) Opposing somatic and dendritic expression of stimulus-selective response plasticity in mouse primary visual cortex. Front Cell Neurosci 13:555.

Kissinger ST, Pak A, Tang Y, Masmanidis SC, Chubykin AA (2018) Oscillatory encoding of visual stimulus familiarity. J Neurosci 38:62236240.

Korotkova T, Fuchs EC, Ponomarenko A, von Engelhardt J, Monyer H (2010) NMDA receptor ablation on parvalbumin-positive interneurons impairs hippocampal synchrony, spatial representations, and working memory. Neuron 68:557-569.

Kuki T, Fujihara K, Miwa H, Tamamaki N, Yanagawa Y, Mushiake H (2015) Contribution of parvalbumin and somatostatin-expressing GABAergic neurons to slow oscillations and the balance in beta-gamma oscillations across cortical layers. Front Neural Circuits 9:6.

Lewis DA, Curley AA, Glausier JR, Volk DW (2012) Cortical parvalbumin interneurons and cognitive dysfunction in schizophrenia. Trends Neurosci 35:57-67.

Luczak A, Bartho P, Harris KD (2013) Gating of sensory input by spontaneous cortical activity. J Neurosci 33:1684-1695.

Makino H, Komiyama T (2015) Learning enhances the relative impact of top-down processing in the visual cortex. Nat Neurosci 18:1116-1122.

Mathis A, Mamidanna P, Cury KM, Abe T, Murthy VN, Mathis MW, Bethge M (2018) DeepLabCut: markerless pose estimation of user-defined body parts with deep learning. Nat Neurosci 21:1281-1289.
Niell CM, Stryker MP (2010) Modulation of visual responses by behavioral state in mouse visual cortex. Neuron 65:472-479.

Pachitariu M, Stringer C, Dipoppa M, Schröder S, Rossi LF, Dalgleish H, Carandini M, Harris KD (2017) Suite2p: beyond 10,000. neurons with standard two-photon microscopy. bioRxiv 061507. doi: 10.1101/061507.

Pakan JM, Lowe SC, Dylda E, Keemink SW, Currie SP, Coutts CA, Rochefort NL (2016) Behavioral-state modulation of inhibition is context-dependent and cell type specific in mouse visual cortex. Elife 5:e14985.

Pfeffer CK, Xue M, He M, Huang ZJ, Scanziani M (2013) Inhibition of inhibition in visual cortex: the logic of connections between molecularly distinct interneurons. Nat Neurosci 16:1068-1076.

Polepalli JS, Wu H, Goswami D, Halpern CH, Südhof TC, Malenka RC (2017) Modulation of excitation on parvalbumin interneurons by neuroligin-3 regulates the hippocampal network. Nat Neurosci 20:219-229.

Reimer J, Froudarakis E, Cadwell CR, Yatsenko D, Denfield GH, Tolias AS (2014) Pupil fluctuations track fast switching of cortical states during quiet wakefulness. Neuron 84:355-362.

Reimer J, McGinley MJ, Liu Y, Rodenkirch C, Wang Q, McCormick DA, Tolias AS (2016) Pupil fluctuations track rapid changes in adrenergic and cholinergic activity in cortex. Nat Commun 7:13289.

Saleem AB, Lien AD, Krumin M, Haider B, Rosón MR, Ayaz A, Reinhold K, Busse L, Carandini M, Harris KD (2017) Subcortical source and modulation of the narrowband gamma oscillation in mouse visual cortex. Neuron 93:315-322.

Saravanan V, Berman GJ, Sober SJ (2020) Application of the hierarchical bootstrap to multi-level data in neuroscience. bioRxiv 819334. doi: $10.1101 / 819334$

Sokolov EN (1963) Higher nervous functions; the orienting reflex. Annu Rev Physiol 25:545-580.

van Vugt MK, Sederberg PB, Kahana MJ (2007) Comparison of spectral analysis methods for characterizing brain oscillations. J Neurosci Methods 162:49-63.

Veit J, Hakim R, Jadi MP, Sejnowski TJ, Adesnik H (2017) Cortical gamma band synchronization through somatostatin interneurons. Nat Neurosci 20:951-959.

Vinck M, Batista-Brito R, Knoblich U, Cardin JA (2015) Arousal and locomotion make distinct contributions to cortical activity patterns and visual encoding. Neuron 86:740-754.

Xu H, Jeong HY, Tremblay R, Rudy B (2013) Neocortical somatostatinexpressing GABAergic interneurons disinhibit the thalamorecipient layer 4. Neuron 77:155-167.

Zhou H, Schafer RJ, Desimone R (2016) Pulvinar-cortex interactions in vision and attention. Neuron 89:209-220. 